Article

\title{
Bloch Modes and Evanescent Modes of Photonic Crystals: Weak Form Solutions Based on Accurate Interface Triangulation
}

\author{
Matthias Saba $^{1, *}$ and Gerd E. Schröder-Turk ${ }^{1,2}$ \\ ${ }^{1}$ Theoretische Physik, Friedrich-Alexander Universität Erlangen-Nürnberg, Staudtstr. 7B, \\ 91058 Erlangen, Germany; E-Mail: gerd.schroeder-turk@fau.de \\ ${ }^{2}$ Applied Maths, Research School of Physical Sciences and Engineering, The Australian \\ National University, Canberra 0200 ACT, Australia \\ * Author to whom correspondence should be addressed; E-Mail: matthias.saba@fau.de; \\ Tel.: +49-9131-85-28452.
}

Academic Editor: Yuri Kivshar

Received: 20 August 2014 / Accepted: 10 December 2014 / Published: 5 January 2015

\begin{abstract}
We propose a new approach to calculate the complex photonic band structure, both purely dispersive and evanescent Bloch modes of a finite range, of arbitrary three-dimensional photonic crystals. Our method, based on a well-established plane wave expansion and the weak form solution of Maxwell's equations, computes the Fourier components of periodic structures composed of distinct homogeneous material domains from a triangulated mesh representation of the inter-material interfaces; this allows substantially more accurate representations of the geometry of complex photonic crystals than the conventional representation by a cubic voxel grid. Our method works for general two-phase composite materials, consisting of bi-anisotropic materials with tensor-valued dielectric and magnetic permittivities $\varepsilon$ and $\mu$ and coupling matrices $\zeta$. We demonstrate for the Bragg mirror and a simple cubic crystal closely related to the Kelvin foam that relatively small numbers of Fourier components are sufficient to yield good convergence of the eigenvalues, making this method viable, despite its computational complexity. As an application, we use the single gyroid crystal to demonstrate that the consideration of both conventional and evanescent Bloch modes is necessary to predict the key features of the reflectance spectrum by analysis of the band structure, in particular for light incident along the cubic [111] direction.
\end{abstract}


Keywords: photonic crystals; band structure; evanescent modes; weak form solutions; single gyroid; reflectance; mesh representations; band gap material

\section{Introduction}

A photonic crystal $(\mathrm{PhC})$ is a composite material with a periodic and symmetric spatial arrangement of at least two components with different macroscopic electromagnetic properties, such as the dielectric constant $\varepsilon$ (or only its real part in the original definition [1,2]) and, more generally, also the magnetic permittivity $\mu$ and the coupling constant $\zeta$ [3]. The photonic band structure represents the dispersion relation between the Bloch wave vector $\mathbf{k}$ and frequency $\omega$ of the periodic electromagnetic field configurations, called Bloch modes, which are commensurate with the periodicity and symmetry of the $\mathrm{PhC}$.

The photonic band structure (PBS) is a concept [2] that is often invoked when one is interested in the reflectance or transmission properties of a slab of photonic crystal, even though the finite nature of the slab breaks the spatial periodicity. The existence of a photonic band gap, i.e., a frequency range for which no Bloch modes exist in the infinite $\mathrm{PhC}$, is commonly interpreted as a stop band, i.e., a frequency interval for which no light can penetrate through the photonic crystal and is, hence, reflected. Similarly, it is often assumed that light with a frequency that matches the frequency of a given Bloch mode may penetrate through the crystal, subject to sufficient coupling of the incident light and the Bloch mode, which acts as a transmission channel.

Simple approaches to evaluate the degree of coupling between an incoming plane wave and the Bloch modes of a PhC suffice in many situations to correlate features of the band structure to the transmission or reflectance spectra [4], e.g., for light incident onto a semi-infinite single gyroid or a four-srs $\mathrm{PhC}$ along the [100] or [110] lattice direction [5]. The single gyroid is a complex network-like ordered $\mathrm{PhC}$ with structural chirality, of relevance both as a biophotonic and as a synthetic material [6]; see Figure 1.

However, the general solution of the problem of the reflectance of a plane wave incident onto a finite or semi-infinite slab of a $\mathrm{PhC}$ is a scattering problem that involves not only the purely dispersive Bloch modes, but also evanescent Bloch modes, whose amplitude decreases away from the interface; see Figure 2. While the bulk modes themselves can be extracted to high accuracy from, e.g., finite element method (FEM) calculations $(c f .[7,8])$, the scattering problem itself is conveniently solved in Fourier space using Floquet's theorem and the orthogonality of plane waves [9-12]. Hence, the accuracy is lost laterally as a result of the numerical Fourier cutoff. We therefore use a similar approach as in [13] and solve the bulk eigenvalue equation also in Fourier space to obtain the generalized Bloch modes, including those that are evanescent. This earlier work [13] did not mention how the Fourier transforms are performed while presumably having the same memory limitation (without mentioning this fact explicitly) due to matrix inversion (see Section 3.2), as the authors show a convergence diagram for a very small number of plane waves $N<1500$ in a two-dimensional case only. If we assume that some kind of discrete Fourier transformation has been done, our algorithm provides roughly one order of magnitude increased accuracy for the same number of plane waves ( $c f$. Section 5.1) and is further more 
general in terms of the material properties: We consider for examaple anisotropy as well as Condon cross-coupling, see constitutive Equations (1) and (2).
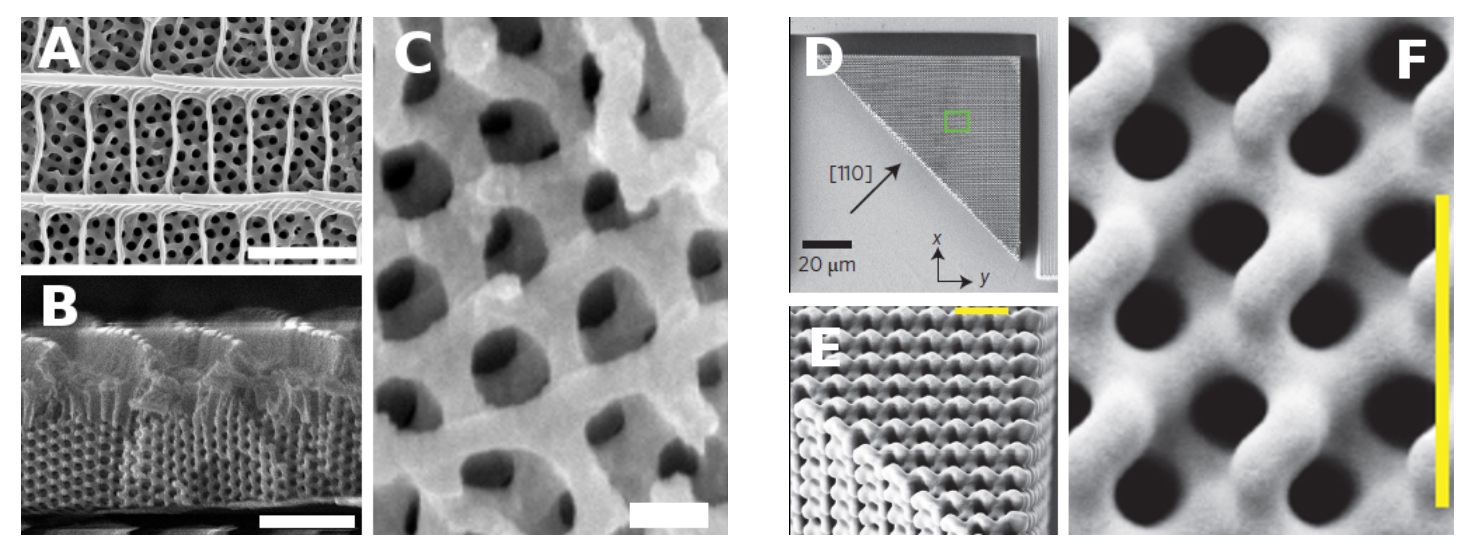

Figure 1. Biological and synthetic realisations of the single-gyroid photonic crystal. (Left) Porous chitin structure in the wing-scales of the butterfly, Callophrys rubi. (A) Top view of wing scale (scale bar: $2 \mu \mathrm{m}$ ); $(\mathbf{B})$ side view (scale bar: $2 \mu \mathrm{m}$ ); $(\mathbf{C})$ top view (scale bar: $200 \mathrm{~nm}$ ). The lattice parameter of the $I 4_{1} 32$ space group is around $a \approx 0.3 \mu \mathrm{m}$ (images adapted from [14]). (Right) Chiral beam-splitter device based on the single gyroid structure with $a=1.2 \mu \mathrm{m}$, produced by galvo-dithered direct laser [15]; (D) perspective view of the prism-shaped device consisting of $80^{2} \times 20 / 2$ translational unit cells; (E) close-up with the scale bar being $2 \mu \mathrm{m}$; (F) top view with the scale bar being $2 \mu \mathrm{m}$ (images adapted from [15], copyright 2013 Nature Publishing Group).

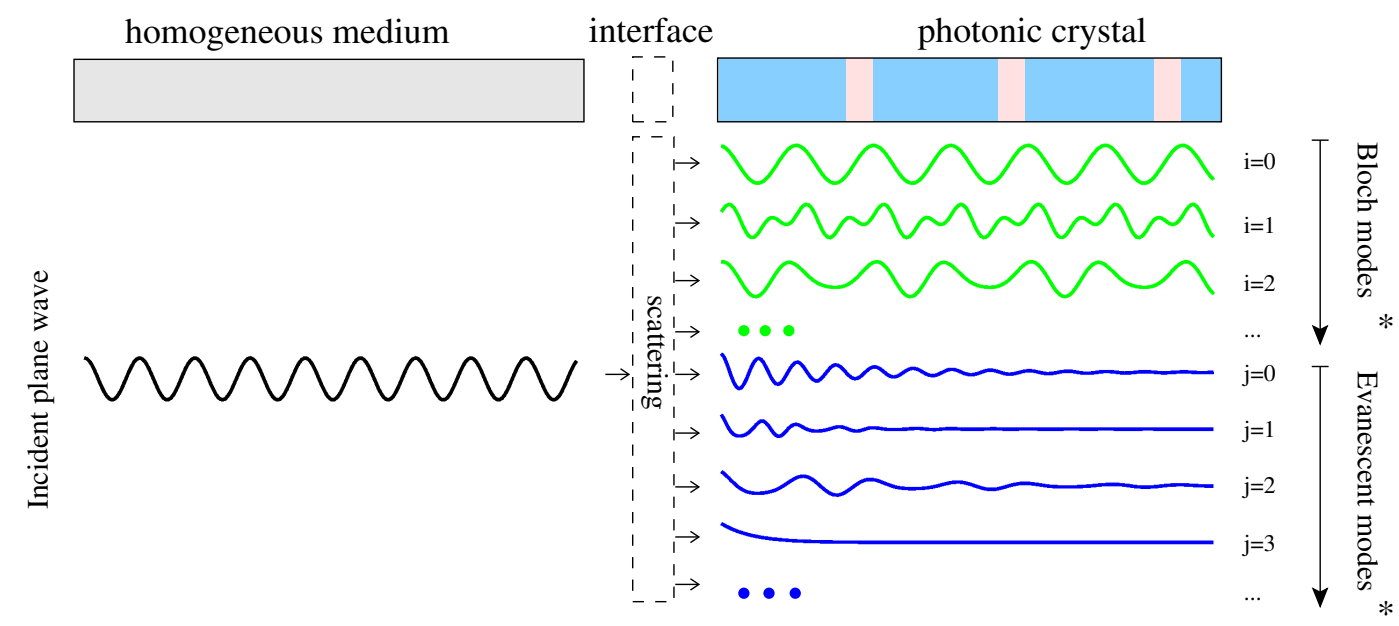

Figure 2. Mock-illustration of the scattering process of a plane wave incident onto a slab of photonic crystal: both conventional dispersive Bloch modes (labelled $i$ ) and evanescent modes (whose amplitude decays away from the interface labelled $j$ ) need to be taken into account when evaluating the reflectance and transmission properties. (*) Note that the display of both the Bloch modes and of the evanescent modes excludes the (aperiodic) phase factor $\exp (\imath k r)$. 
While a slow geometrical convergence is part of any numerical Fourier approach for piecewise continuous functions due to the Gibbs phenomenon (a truncated Fourier sum overshoots the original function at discontinuities) and cannot be entirely avoided [16], our approach allows one to compute the components of the material matrices with machine precision avoiding staircase effects, which are a problem in the standard approach [17] based on a regular spatial lattice that is well known to yield additional numerical errors, $c f$. [18-20] for the finite difference time domain (FDTD) methods. Figure 3 illustrates the representation of a complex three-dimensional $\mathrm{PhC}$, the same single gyroid structure as shown in Figure 1, by voxels (Figure 3b) and by a mesh (Figure 3c) and the apparent advantage of the latter. One of the contributions of this article is the demonstration that the complex photonic band structure can be directly computed from a mesh representation, hence eliminating the error due to conventional discrete Fourier transformations that is particularly large for a small number of Fourier components, compared to error related to the Gibbs phenomenon.

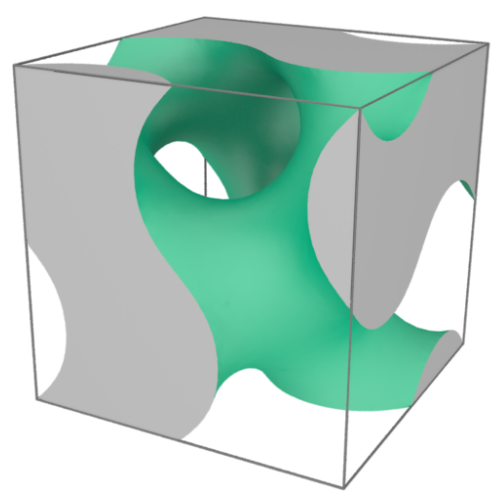

(a)

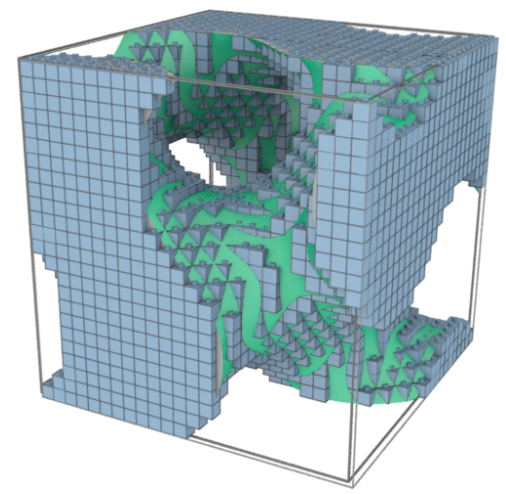

(b)

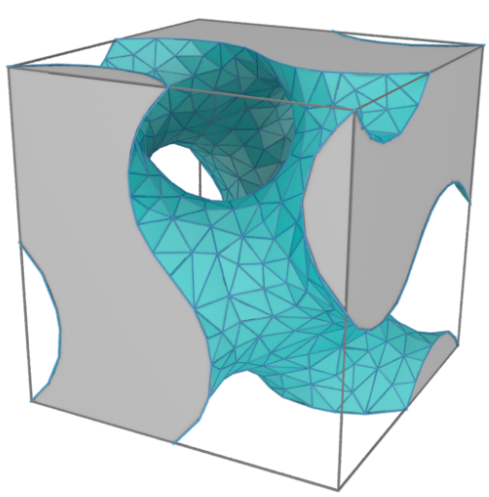

(c)

Figure 3. Mesh and voxel representation of photonic crystals with a single gyroid structure: (a) the single gyroid geometry [21,22], which has attracted recent attention as a photonic material [5,23-27], is a two-phase geometry with cubic symmetry with a solid (gray) and a void (transparent) component, each of which forms a labyrinth- or network-like component; (b) a commonly-used representation (e.g., in the MIT packages MEEP [28] or MBP [17]) is the voxelised "LEGO ${ }^{\circledR}$-like" representation, where space is represented by a regular voxel grid with each voxel being either solid or void; this representation always leads to stair-case errors; see, in particular, the Appendix of [18]; (c) a representation of the geometry by a mesh (or triangulation) representing the interface between solid and void yields more accurate geometric representations.

\section{Generalized Bloch Mode Equation}

In this section, the differential eigenvalue equation that yields the Bloch modes is derived: the bases are Maxwell's equations in the monochromatic form with a fixed frequency $\omega=c k_{0}$ (with the vacuum speed of light $c$ and the vacuum wave number $k_{0} \in \mathbb{R}$ ) and lateral wave vector $\mathbf{k}_{t} \in \mathbb{R}^{2}$ (or, equivalently, the angle of incidence). Each Bloch mode that is able to couple to an incident plane wave is thus characterized by the wave number $k_{z} \in \mathbb{C}$ in the surface normal direction, which plays the role of an eigenvalue. 
Here, and in the following, the lattice constant of the $\mathrm{PhC}$ is set to $a=1$, so that electromagnetic fields (or, more precisely, the complex spatial amplitudes in a monochromatic approach) are considered as functions $\mathbf{E}: \mathbb{R}^{3} \rightarrow \mathbb{C}^{3}$, etc., i.e., we omit the spatial dimension. As a result, all wave numbers are also dimensionless. In practice, scaling with the lattice constant yields the physical solution from this slightly more abstract formulation.

\subsection{Material Parameters and Maxwell's Equations}

The $\mathrm{PhC}$ is assumed to be a combination of linear bi-anisotropic materials that satisfy Lorentz-reciprocity [3]. The corresponding constitutive equations have the following general form:

$$
\begin{aligned}
& \mathbf{D}=\underline{\underline{\varepsilon}} \mathbf{E}+\underline{\underline{\zeta}} \mathbf{H} \\
& \mathbf{B}=\underline{\underline{\mu}} \mathbf{H}-\underline{\underline{\zeta}} \mathbf{E}
\end{aligned}
$$

These two equations relate the displacement field $\mathbf{D}$ and the magnetic flux $\mathbf{B}$ to the electric field $\mathbf{E}$ and the magnetic field $\mathbf{H}$, respectively. The microscopic physics is described by the symmetric permittivity and permeability matrices $\underline{\underline{\varepsilon}}$ and $\underline{\mu}$ and the coupling matrix $\underline{\zeta}$, all $\in \mathrm{GL}_{3}(\mathbb{C})$. (Note that these assumptions of the material properties of the constituent materials are rather broad, encompassing lossy and anisotropic materials with a spatially varying orientation of the permittivity and permeability matrices and bi-isotropic materials with coupling between electric and magnetic contributions to the displacement field and the magnetic flux. The scalar case goes back to Condon [29], where the coupling $\zeta$ is directly related to the chirality $\kappa$ via $\zeta=\imath \sqrt{\varepsilon \mu} \kappa$ [30]. Evidently, the standard case of a $\mathrm{PhC}$ made of an isotropic dielectric material is covered by this formalism as $\underline{\underline{\varepsilon}}=\varepsilon \mathbb{1}, \underline{\underline{\mu}}=\mathbb{1}$ and $\zeta=0$.) Note that the material parameters may also be frequency dependent without further restriction in our algorithm. For the sake of clearer nomenclature, we have restricted the notation to frequency-independent material properties.

The macroscopic Maxwell equations in the monochromatic form are given by (with $k_{0}=\omega / c$ the vacuum wave number and using conventional Gaussian units [31]) (The divergence equations, i.e., the Coulomb and the Gauss law, are redundant here; due to the Poincaré lemma [32] for a differential two-form in $\mathbb{R}^{3}$, i.e., $\nabla \cdot \mathbf{F}=0 \Leftrightarrow \mathbf{F}=\nabla \times \mathbf{A}$ for some $\mathbf{A}$.):

$$
\begin{aligned}
\nabla \times \mathbf{E} & =\imath k_{0} \mathbf{B} \\
\nabla \times \mathbf{H} & =-\imath k_{0} \mathbf{D}
\end{aligned}
$$

Equations (3) and (4) have the form of a system of six linear first-order partial differential equations that can be written as:

$$
\theta \mathbf{F}=\imath k_{0} \mathcal{M} \mathbf{F}
$$

with the field vector $\mathbf{F}=\left(\mathbf{H}^{T}, \mathbf{E}^{T}\right)^{T}$; the superscript $(\cdot)^{T}$ denotes the transpose operation. The frequency-dependent differential operator matrix $\theta$ and the material matrix $\mathcal{M}$ are:

$$
\theta=\left(\begin{array}{cc}
0 & \text { curl } \\
\text { curl } & 0
\end{array}\right), \quad \operatorname{curl}=\left(\begin{array}{ccc}
0 & -\partial_{z} & \partial_{y} \\
\partial_{z} & 0 & -\partial_{x} \\
-\partial_{y} & \partial_{x} & 0
\end{array}\right) ; \quad \mathcal{M}=\left(\begin{array}{cc}
\underline{\mu} & -\underline{\underline{\zeta}} \\
-\underline{\underline{\zeta}} & -\underline{\underline{\varepsilon}}
\end{array}\right)
$$




\subsection{Geometry}

We consider PhCs composed of two types of material (indexed by zero and one), whose spatial distribution is given by the indicator function $\chi: \Omega \rightarrow\{0,1\}$ that takes the value $\chi(\mathbf{r})=\alpha$ for a given point $\mathbf{r}$ that is occupied by material with $\alpha=0,1$. The $\mathrm{PhC}$ and, hence, $\chi$ are periodic, and we denote the three basis vectors of the PhC lattice as $\mathbf{a}_{i} \in \mathbb{R}^{3}$. The unit cell $\Omega \subset \mathbb{R}^{3}$ of the $\mathrm{PhC}$ is the parallelepiped spanned by these basis vectors. It is a composition $\Omega=\Omega_{0} \cup \Omega_{1}$ of two spatial domains $\Omega_{\alpha}=\{\mathbf{r} \in \Omega \mid \chi(\mathbf{r})=\alpha\}$ that are separated by an interfacial surface $S=\partial \Omega_{1} \cap \partial \Omega_{2}$. (Note that the interfacial surface $S$ is not necessarily represented by a single connected component. For example, a Bragg mirror has two non-connected planar surfaces between its two domains; Section 5.1.) Each material function can be expressed by $f(\mathbf{r})=f_{0}+\left(f_{1}-f_{0}\right) \chi(\mathbf{r})$ with constant parameters $f_{\alpha}\left(f=\varepsilon_{i j}, \mu_{i j}, \zeta_{i j} ; i, j=1,2,3\right)$.

\subsection{The Wave Vector Eigenvalue Equation}

We now develop the operators that allow one to express the problem of solving the partial differential Equation (5) in a weak formulation, i.e., as a solution of a corresponding integral equation with lesser differentiability requirements. This approach, which provides the general basis for finite element methods [33] and which is developed in this section for the spatially continuous situation, lends itself to a discretization by simplices, i.e., triangulations, rather than discretizing the volume into voxels, avoiding systematic errors in the numerical approximation of the original structure, such as symmetry breaks (see the Appendix of [18] as an example) and the introduction of sharp interface angles.

We solve Equation (5) for all bulk $\mathrm{PhC}$ modes that can couple to an incoming plane wave with wave vector $\mathbf{q}=\left(k_{x}, k_{y}, \sqrt{k_{0}^{2}-k_{x}^{2}-k_{y}^{2}}\right)^{T} \in \mathbb{R}^{2} \times \mathbb{C}$. The $z$-direction coincides with the inclination direction of the semi-infinite or slab-like PhC structure. Due to the spatial periodicity and Floquet's theorem, the fields that solve Equation (5) are elements of the linear solution space with basis functions:

$$
\mathbf{E}(\mathbf{r})=\hat{\mathbf{E}}(\mathbf{r}) e^{\imath \mathbf{k} \cdot \mathbf{r}} ; \quad \hat{\mathbf{E}}(\mathbf{r}+\mathbf{T})=\hat{\mathbf{E}}(\mathbf{r})
$$

and equivalent for $\mathbf{H}$, with lattice vector $\mathbf{T}$, i.e., any linear combination of the $\mathbf{a}_{i}$. The Floquet exponents are given by $\imath k_{i}$ with the vanishing real part for $i=x, y$. The real part is further less than zero (Lyapunov stability) for $i=z$ if the $\mathrm{PhC}$ is semi-infinite. The physical reason for these restrictions is due to the fact that we do not consider solutions for which the field energy density diverges at infinity.

The vector $\mathbf{k}=\left(k_{x}, k_{y}, k_{z}\right)^{T} \mathbb{R}^{2} \times \mathbb{C}$ is referred to as the Bloch wave vector, although, in contrast to classical Bloch modes, $k_{z} \in \mathbb{C}$ is complex in general. (The wave numbers $k_{x}, k_{y}$ parallel to the interface are conserved as a result of the boundary conditions, which becomes immediately evident in Fourier space. From a group theoretical perspective, one could also argue that the solution in the whole space is laterally of the Bloch form as a result of the periodicity, and the phase factor $\exp \left\{\imath \mathbf{k}_{\|} \cdot \mathbf{T}_{\|}\right\}$can be interpreted as the character of a set of infinite one-dimensional irreducible representations that are all orthogonal to each other; $c f$. [18]).

We rearrange Equation (5) to convert it into the following form:

$$
K \mathbf{v}=k_{z} M \mathbf{v}
$$


where $\mathbf{v}(\mathbf{r})$ contains the periodic part of the fields:

$$
\mathbf{v}=\left(\begin{array}{c}
\hat{\mathbf{H}}(\mathbf{r}) \\
\hat{\mathbf{E}}(\mathbf{r})
\end{array}\right)
$$

and the operators are given by:

$$
K=\left(\begin{array}{cc}
-\underline{\underline{\mu}} & \underline{\underline{\zeta}}+k_{0}^{-1} \underline{\underline{k}}+\left(\imath k_{0}\right)^{-1} \operatorname{curl} \\
\underline{\underline{\zeta}}+k_{0}^{-1} \underline{\underline{k}}+\left(\imath k_{0}\right)^{-1} \operatorname{curl} & \underline{\underline{\varepsilon}}
\end{array}\right) ; \quad M=\left(\begin{array}{ll}
\underline{\underline{0}} & \frac{\underline{I}}{\underline{\underline{\underline{\theta}}}}
\end{array}\right)
$$

where the three-dimensional matrices are defined by:

$$
\underline{\underline{k}}=\left(\begin{array}{ccc}
0 & 0 & k_{y} \\
0 & 0 & -k_{x} \\
-k_{y} & k_{x} & 0
\end{array}\right) ; \quad \underline{\underline{I}}=\frac{1}{k_{0}}\left(\begin{array}{ccc}
0 & 1 & 0 \\
-1 & 0 & 0 \\
0 & 0 & 0
\end{array}\right)
$$

The rank of the matrix $M$ in Equation (11) is four, while its dimension is six, leading to spurious modes polarized in the $z$-direction and with an infinite eigenvalue. Reduction to a wave equation as in [34] yields a full second order eigenvalue equation just for one of the fields $\mathbf{E}$ or $\mathbf{H}$ for $\zeta \neq 0$, which is of same complexity as the original linear form presented here.

Instead, we eliminate the $z$ components of the fields, as, for example, done in $[11,12]$, to obtain a reduced problem of dimension four. We first introduce block-matrices:

$$
\begin{aligned}
& K_{\|}=\left(\begin{array}{cccc}
-\mu_{11} & -\mu_{12} & \zeta_{11} & \zeta_{12}-\frac{1}{\imath k_{0}} \partial_{z} \\
-\mu_{21} & -\mu_{22} & \zeta_{21}+\frac{1}{\imath k_{0}} \partial_{z} & \zeta_{22} \\
\zeta_{11} & \zeta_{12}-\frac{1}{\imath k_{0}} \partial_{z} & \varepsilon_{11} & \varepsilon_{12} \\
\zeta_{21}+\frac{1}{\imath k_{0}} \partial_{z} & \zeta_{22} & \varepsilon_{21} & \varepsilon_{22}
\end{array}\right) \\
& K_{\perp}=\left(\begin{array}{cc}
-\mu_{33} & \zeta_{33} \\
\zeta_{33} & \varepsilon_{33}
\end{array}\right) \\
& K_{\| \rightarrow \perp}=\left(\begin{array}{cc}
-\mu_{13} & \zeta_{13}+\frac{k_{y}-\imath \partial_{y}}{k_{0}} \\
-\mu_{23} & \zeta_{23}-\frac{k_{x}-\imath \partial_{x}}{k_{0}} \\
\zeta_{13}+\frac{k_{y}-\imath \partial_{y}}{k_{0}} & \varepsilon_{13} \\
\zeta_{23}-\frac{k_{x}-\imath \partial_{x}}{k_{0}} & \varepsilon_{23}
\end{array}\right) \\
& K_{\perp \rightarrow \|}=\left(\begin{array}{cccc}
-\mu_{31} & -\mu_{32} & \zeta_{31}-\frac{k_{y}-\imath \partial_{y}}{k_{0}} & \zeta_{32}+\frac{k_{x}-\imath \partial_{x}}{k_{0}} \\
\zeta_{31}-\frac{k_{y}-\imath \partial_{y}}{k_{0}} & \zeta_{32}+\frac{k_{x}-\imath \partial_{x}}{k_{0}} & \varepsilon_{31} & \varepsilon_{32}
\end{array}\right)
\end{aligned}
$$

and corresponding vectors:

$$
\mathbf{v}_{\|}=\left(\mathbf{v}_{1}, \mathbf{v}_{2}, \mathbf{v}_{4}, \mathbf{v}_{5}\right)^{T} ; \quad \mathbf{v}_{\perp}=\left(\mathbf{v}_{3}, \mathbf{v}_{6}\right)^{T}
$$

and analogous for $M$, so that $M_{\perp}=0, M_{\| \rightarrow \perp}=0$ and $M_{\perp \rightarrow \|}=0$. Equation (7) thus yields the four-dimensional eigenvalue equation:

$$
S^{-1} M_{\|} \mathbf{v}_{\|}=k_{z}^{-1} \mathbf{v}_{\|}
$$


with the Schur complement $S:=K_{\|}-K_{\| \rightarrow \perp} K_{\perp}^{-1} K_{\perp \rightarrow \|}$, for which the inverse of $K_{\perp}$ is given by:

$$
K_{\perp}^{-1}=\frac{1}{\varepsilon_{33} \mu_{33}+\zeta_{33}^{2}}\left(\begin{array}{cc}
-\varepsilon_{33} & \zeta_{33} \\
\zeta_{33} & \mu_{33}
\end{array}\right)
$$

\subsection{The Weak Form of the Eigenvalue Problem}

We now convert Equation (10) into a weak form that is discretized in the following section. We define the Banach space $V$ of functions $f: \Omega \rightarrow \mathbb{C}$ that map the translational unit cell $\Omega$ onto the complex plane. (More precisely, $V$ is a special type of Sobolev space for which we need to assume square integrability for differentiating up to second order. Furthermore, we need to assume periodicity in the sense that on the boundary of $\Omega, f(\mathbf{r})=f(\mathbf{r}+\mathbf{T})$ and also for the derivative of $f$. However, with a plane wave expansion, our discretized Galerkin space naturally meets both restrictions. In fact, infinite differentiability is a too strong of a requirement that leads to the well-known Gibbs phenomenon [16].) The corresponding norm for $v \in V$ is $(v, v)^{1 / 2}$ with the sesquilinear form $(v, w)=\int_{\Omega} d^{3} r v^{*} w$, where $(\cdot)^{*}$ denotes complex conjugation and $d^{3} r=d r_{1} d r_{2} d r_{3}$ the volume element. More generally, the sesquilinear form for $\mathbf{v}, \mathbf{w} \in V^{n}$ is $(\mathbf{v}, \mathbf{w})=\int_{\Omega} d^{3} r \mathbf{v} \cdot \mathbf{w}$ with the $n$-dimensional scalar product defined by $\mathbf{v} \cdot \mathbf{w}:=\sum_{i=1}^{n} v_{i}^{*} w_{i}$. Using those definitions, the reduced strong problem Equation (10) has the following weak form:

$$
\text { Find } \mathbf{v}_{\|} \in V^{4} \text { so that } \forall \mathbf{u} \in V^{4}: \quad\left(\mathbf{u}, S^{-1} M_{\|} \mathbf{v}_{\|}\right)=k_{z}^{-1}\left(\mathbf{u}, \mathbf{v}_{\|}\right)
$$

All solutions of Equation (11) thus form a complete set of basis functions (see Floquet's theorem above) for the solution of Equation (5), for fixed lateral dispersion and physical boundary conditions at infinity (see the previous Section 2.3.)

\section{Plane Wave Expansion}

All plane waves that meet the periodicity requirement serve as a complete basis set for the Banach space $V$ and may hence be used as test functions in each of the components of $\mathbf{u}$ in Equation (11):

$$
u_{\mathbf{G}}^{(i)}(\mathbf{r})=e^{-\imath \mathbf{G} \cdot \mathbf{r}} \mathbf{e}_{i}
$$

Here, we introduce the unit vector $\mathbf{e}_{i}$, whose $i$-th component is one, and any other vanishes, and the reciprocal lattice vectors $\mathbf{G} \in \mathcal{G}=\left\{B \cdot \mathbf{l} \mid \mathbf{l} \in \mathbb{Z}^{3}\right\}$, where $B$ is the reciprocal basis matrix $B=2 \pi\left(A^{T}\right)^{-1}$, that is, the transposed inverse of the lattice matrix $A=\left(\mathbf{a}_{1}, \mathbf{a}_{2}, \mathbf{a}_{3}\right)$. The field vector $\mathbf{v}$ is expanded by the same plane wave basis and substituted by its Fourier series:

\subsection{Fourier Integrals}

$$
\mathbf{v}(\mathbf{r})=\sum_{\mathbf{G} \in \mathcal{G}} e^{-\imath \mathbf{G} \cdot \mathbf{r}} \mathbf{v}_{\mathbf{G}}
$$

The Fourier-Galerkin equation is composed of integrals of the form $\int_{\Omega} d^{3} r f(\mathbf{r}) \exp \{\imath(\mathbf{G}) \cdot \mathbf{r}\}$, where $f(\mathbf{r})$ is either a material function or a constant. In this section, we derive a method to calculate these Fourier integrals up to machine precision using a triangulation of the interfacial surface $S$. It corresponds 
to an analogous method that has been developed in two dimensions (Lemma 5.4, the direct method in [35]). (Sorets [35] also describes a way to substantially decrease the computational cost compared to the direct method using Gaussian quadratures and the fast Fourier transform. As the calculation of the Fourier integrals is not time critical for our purpose (it scales with $n^{2}$ if $n$ is the number of nodes of the triangulation and is done once for a given geometry), we do not use this generally very useful idea here.)

We use the expression $f(\mathbf{r})=f_{0}+\Delta f \chi(\mathbf{r})$ (with the indicator function $\chi(\mathbf{r})$ and $\Delta f:=f_{1}-f_{0}$; Section 2.2) to calculate the integral integral over the unit cell:

$$
(\mathcal{F} f)_{\mathbf{G}}:=\int_{\Omega} d^{3} r f(\mathbf{r}) e^{\imath \mathbf{G} \cdot \mathbf{r}}=f_{0} \int_{\Omega} d^{3} r e^{\imath \mathbf{G} \cdot \mathbf{r}}+\Delta f \int_{\Omega_{1}} d^{3} r e^{\imath \mathbf{G} \cdot \mathbf{r}}
$$

It is convenient to introduce lattice coordinates $\mathbf{r}_{L}$, defined by $A \mathbf{r}_{L}=\mathbf{r}$ (with the lattice matrix $A$ ), for which the unit cell transforms into the unit cube $\Omega_{L}:=[0,1)^{3}=A^{-1} \Omega$. Note that we are not constrained to a cubic (or orthorhombic) lattice system; any crystal system is valid and equally simple to treat. The Jacobi determinant for the linear transformation into lattice coordinates is simply the volume of the original unit cell $V_{\Omega}=\int_{\Omega} d^{3} r$. The scalar product in the exponent transforms according to $\mathbf{G} \cdot \mathbf{r}=\mathbf{G} \cdot A \mathbf{r}_{L}=A^{T} \mathbf{G} \cdot \mathbf{r}_{L}=2 \pi B^{-1} \mathbf{G} \cdot \mathbf{r}_{L}=2 \pi \mathbf{l} \cdot \mathbf{r}_{L}$ and the Fourier transformation is given by (if $f(\mathbf{r})$ is constant $\left(f_{0}=f_{1}\right)$, this equation simplifies to the trivial expression $(\mathcal{F} f)_{\mathbf{l}=0}=f_{0} V_{\Omega}$ and $\left.(\mathcal{F} f)_{\mathbf{l} \neq 0}=0(c f .[36])\right)$ :

$$
(\mathcal{F} f)_{\mathbf{l}}=V_{\Omega}\left(f_{0} \int_{\Omega_{L}} d^{3} r e^{2 \pi \imath \mathbf{l} \cdot \mathbf{r}}+\Delta f \int_{\Omega_{1}^{(L)}} d^{3} r e^{2 \pi \imath \mathbf{l} \cdot \mathbf{r}}\right)
$$

The integrals in in this equation may be solved analytically in special cases. Generally, we assume that the surface is given by a triangulation $S_{L}=\left\{\Delta_{\tau} \mid \tau=1,2, \ldots, N_{\bowtie}\right\}$; see Figure 4. Recursive application of Stokes' theorem in Euclidean $\mathbb{R}^{d}$ and for a differential $d-1$-form $\omega$ allows one to express the volume integrals as a sum over the vertices of the triangulated surface $S_{L}$ : In particular, we start with the divergence theorem in $d=3$ that yields facet integrals and apply the divergence theorem in $d=2$ onto each facet (equivalent to Green's theorem) to obtain line integrals that are evaluated at their end points (divergence theorem in $d=1$, aka the second fundamental theorem of calculus). We introduce local vectors for the triangle $\tau$ with edges $\boxminus_{\tau \eta}(\eta=1,2,3)$ indexed in a right rotating sense, as seen from region $\Omega_{0}^{(L)}=A^{-1} \Omega_{0}=\Omega_{L} \backslash \Omega_{1}^{(L)}$; see Figure $5 \mathrm{~b}$ :

1. Edge end points:

2. Edge centre points:

3. Edge end-to-end vector:

4. Triangle normal vector:

5. Triangle unit normal:

6. Edge normal vector:

$$
\begin{aligned}
& \mathbf{r}_{\tau \eta}^{( \pm)} \\
& \overline{\mathbf{r}}_{\tau \eta}=\left(\mathbf{r}_{\tau \eta}^{(+)}+\mathbf{r}_{\tau \eta}^{(-)}\right) / 2 \\
& \delta \mathbf{r}_{\tau \eta}=\mathbf{r}_{\tau \eta}^{(+)}-\mathbf{r}_{\tau \eta}^{(-)} \\
& \mathbf{p}_{\tau}=\sum_{\eta=1}^{3} \mathbf{r}_{\tau \eta}^{(-)} \times \mathbf{r}_{\tau \eta}^{(+)} \\
& \mathbf{n}_{\tau}=\frac{\mathbf{p}_{\tau}}{p_{\tau}} \text { with } r:=|\mathbf{r}| \text { for any } \mathbf{r} \in \mathbb{R}^{3} \\
& \mathbf{p}_{\tau \eta}=\delta \mathbf{r}_{\tau \eta} \times \mathbf{n}_{\tau}
\end{aligned}
$$



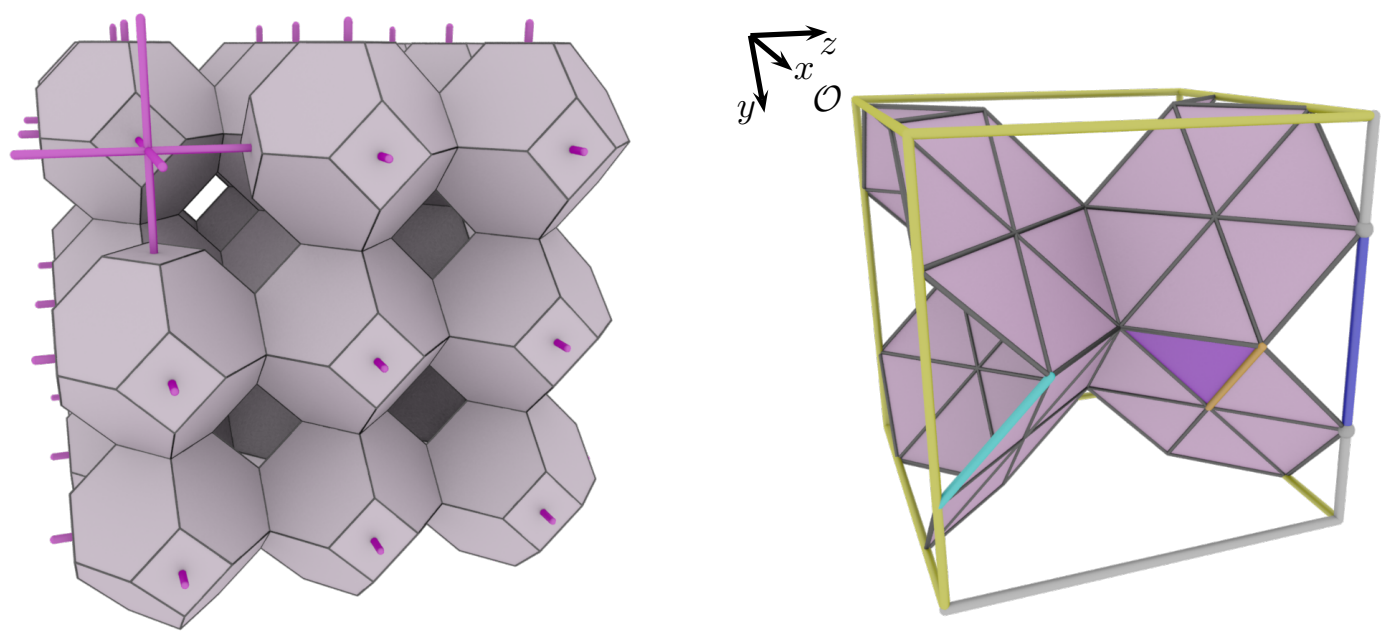

Figure 4. Triangulated representation of the solid/void interfacial surface illustrated for a photonic crystal based on truncated octahedra. Both domains $\Omega_{1}$ and $\Omega_{2}$ are given by truncated octahedra, that is 14-sided polytopes composed of eight regular hexagons and six squares [37]. The chosen unit cell $\Omega\left(=\Omega_{L}\right.$ in this case) is the cube with yellow edges. The origin at the upper left corner of the cube is marked by $\mathcal{O}$. The edges of the triangulation of $S=S_{L}$ are shown in black, and we have picked an arbitrary triangle $\Delta_{\tau}$ over which the sum in Equation (13) goes with one of its three edges $\square_{\tau \eta}$ marked in brown; see the sum in Equation (14). One of the four edges in $\mathcal{E}_{j}$ in Equation (13) is highlighted in turquoise. The distances $d_{j k}$ in Equation (13) are illustrated as follows: the grey bar in the $z$-direction has length $d_{x y}=1 ; d_{x z}=d_{y z}=0.5$ along the other directions, for which half of the edge is in Region 0 (shown in blue for $d_{x z}$ ).

We first calculate the volume integral for the case that $\mathbf{l}=0$ in Equation (12) as a sum over the triangles $\triangle_{\tau}$ and the area of the planar surface $\partial \Omega_{j}=\partial \Omega_{1}^{(L)} \cap\left\{\mathbf{r} \in \mathbb{R}^{3} \mid r_{j}=1\right\}$ that is a boundary of both the region $\Omega_{1}^{(L)}$ and the $j$-th face $\left\{\mathbf{r} \in \mathbb{R}^{3} \mid r_{j}=1\right\}$ of the unit cube (note that integrand vanishes for the remaining three cube faces with $r_{j}=0$ ). $\partial \Omega_{j}$ corresponds to the gray surface domain in Figure $3 \mathrm{c}$. The set of edges $\mathcal{E}_{j}=\left\{\boxminus_{\tau \eta} \mid\left(\overline{\mathbf{r}}_{\tau \eta}\right)_{j}=1\right\}$ that represent part of the boundary of $\partial \Omega_{j}$ is used in the following. Using the Stokes' recurrence procedure described above, the volume integral over $\Omega_{1}^{(L)}$ reduces to:

$$
\begin{aligned}
\int_{\Omega_{1}^{(L)}} d^{3} r & =\frac{1}{3} \int_{\Omega_{1}^{(L)}} d^{3} r \nabla \cdot \mathbf{r} \\
& =\frac{1}{6}\left[\sum_{\tau} \mathbf{r}_{\tau 1}^{(+)} \cdot\left(\mathbf{r}_{\tau 2}^{(+)} \times \mathbf{r}_{\tau 3}^{(+)}\right)+\sum_{j=1}^{3} \mathbf{e}_{j} \cdot\left(\sum_{\nu \in \mathcal{E}_{j}} \mathbf{r}_{\nu}^{(+)} \times \mathbf{r}_{\nu}^{(-)}\right)+\sum_{k \neq j} d_{j k}\right]
\end{aligned}
$$

where $d_{j k}$ is the length of the part of the unit cube's edge $\left\{\mathbf{r} \in \mathbb{R}^{3} \mid r_{j}=1 \wedge r_{k}=1, j \neq k\right\}$ that intersects with the boundary $\partial \Omega_{1}^{(L)}$. Note that the triangulation is clipped to the unit cube by the definition of $S$ in Section 2.2; see also Figure 3. The two vertices $\mathbf{r}_{\nu}^{( \pm)}$of each edge $\square_{\nu}$ are defined by the adjacent triangle $\triangle_{\tau} ; c f$. Figure $5 \mathrm{~b}$. 


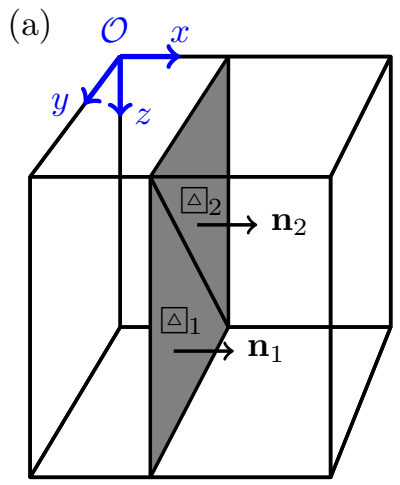

(b)

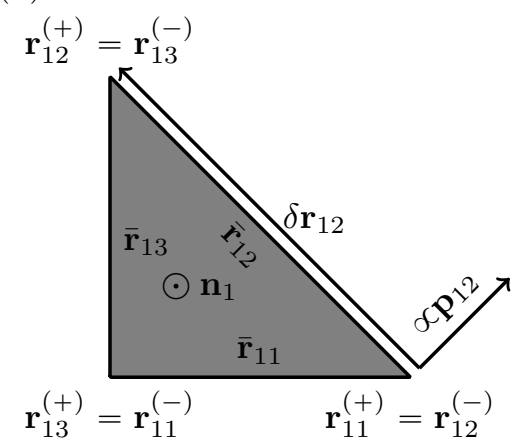

(c)

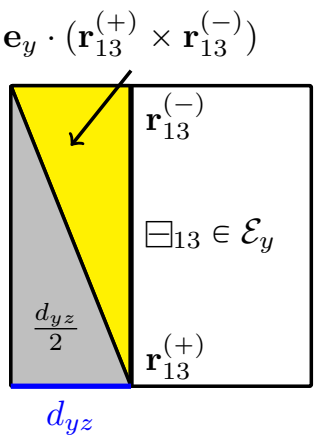

Figure 5. (a) Illustration of a simple triangulated surface $S_{L}$ with normal $\mathbf{n}=\mathbf{e}_{x}$ in the unit cube $\Omega_{L}$. Note that this example serves only to illustrate the definitions used in Section 3.1; it is not periodic. A Bragg mirror ( $c f$. Section 5.1) is defined by two anti-parallel planes. (b) $\Delta_{1}$ as seen from region $\Omega_{L}^{(0)}$ with edge centres $\overline{\mathbf{r}}_{1 \eta}$, end points $\mathbf{r}_{1 \eta}^{( \pm)}$, the exemplary end-to-end vectors $\delta \mathbf{r}_{12}$ and the perpendicular vector (not to scale) $\mathbf{p}_{12}$. (c) Front facet of the unit cube in (a). The surface integral over $\partial \Omega_{y}$ splits up into the yellow part that is calculated via the edge $\square_{13} \in \mathcal{E}_{y}$ corresponding to the second sum in Equation (13) and the light gray part that is calculated with the length $d_{y z}$ of the part of the edge $y=z=1$ that is part of $\partial \Omega_{j}$. The area parts shown here contribute to the volume integral as triangular base faces of pyramids. The volume of the internal pyramids with base faces $\Delta_{1}$ and $\Delta_{2}$, respectively, is calculated in the first sum in Equation (13).

We now reduce Equation (12) for all other cases $l \neq 0$ for which the first term vanishes trivially. The integral in the second term is in the first reduction step written as a sum over the triangles $\Delta_{\tau}$ using the scaled lattice vector $s_{1}=1 /(2 \pi \imath l \cdot l)$ :

$$
\int_{\Omega_{1}^{(L)}} d^{3} r e^{2 \pi \imath \mathbf{l} \cdot \mathbf{r}}=\int_{\Omega_{1}^{(L)}} d^{3} r \nabla \cdot \mathbf{s}_{\mathbf{l}} e^{2 \pi \imath \mathbf{l} \cdot \mathbf{r}}=\sum_{\tau} \mathbf{s}_{\mathbf{l}} \cdot \mathbf{n}_{\tau} \int_{\bigotimes_{\tau}} d^{2} r_{\tau}^{\|} e^{2 \pi \imath \mathbf{l} \cdot \mathbf{r}}
$$

The vector $\mathbf{r}$ is split into its normal component $r_{\tau}^{\perp}=\mathbf{r} \cdot \mathbf{n}_{\tau}$ and its tangential part $\mathbf{r}_{\tau}^{\|}=\mathbf{r}-r_{\tau}^{\perp} \mathbf{n}_{\tau}$ for each $\Delta_{\tau}$; and similar for $\mathbf{l}$. Following the Stokes' reduction procedure, again distinguishing cases $\mathbf{l}_{\tau}^{\|}=0$ and $\mathbf{l}_{\tau}^{\|} \neq 0$, the volume integral finally reduces to:

$$
\int_{\Omega_{1}^{(L)}} d^{3} r e^{2 \pi \imath \mathbf{l} \cdot \mathbf{r}}=\sum_{\tau} \mathbf{s}_{\mathbf{l}} \cdot \mathbf{n}_{\tau} \begin{cases}\frac{p_{\tau}}{2} e^{2 \pi \imath l_{\tau}^{\perp} r_{\tau}^{\perp}} & , \text { if } \mathbf{l}_{\tau}^{\|}=0 \\ \sum_{\eta}\left(\mathbf{s}_{\mathbf{l}_{\tau}^{\|}} \cdot \mathbf{p}_{\tau \eta}\right) e^{2 \pi \imath \mathbf{l} \cdot \overline{\mathbf{r}}_{\tau \eta}} \operatorname{sinc}\left(\pi \mathbf{l} \cdot \delta \mathbf{r}_{\tau \eta}\right) & , \text { else }\end{cases}
$$

with Definitions 1-6 above and the cardinal sine function $\operatorname{sinc}(x)=\sin (x) / x$.

\subsection{The Discrete Problem with a Finite Number of Basis Wave Functions}

The mapping from the continuous to the Bloch form is described by the action of the Bloch-Galerkin operator $\mathcal{B}$ that produces $N \times N$ Bloch matrices according to:

$$
\begin{aligned}
(\mathcal{B} f)_{n m} & =(\mathcal{F} f)_{B^{-1}\left(\mathbf{G}_{m}-\mathbf{G}_{n}\right)} \\
\left(\mathcal{B} \partial_{i}\right)_{n m} & =\imath G_{m}^{(i)}
\end{aligned}
$$


where $n, m \in\{1,2, \ldots, N\}$ and $f$ is any material function. Defining the operator $\mathcal{B}$ so that it acts entry-wise onto the component matrices, i.e., $(\mathcal{B} S)_{i j}=\mathcal{B} S_{i j}$, the algebraic matrices are given by the compact notation:

$$
S_{N}=\mathcal{B} S ; \quad M_{N}=\mathcal{B} M
$$

with $S$ and $M$ defined in Equations (8) and (10).

Equation (11) transforms into the generalized algebraic eigenvalue equation:

$$
\left(S^{-1}\right)_{N}\left(M_{\|}\right)_{N}\left(\mathbf{v}_{\|}\right)_{N}=k_{z}^{-1}\left(\mathbf{v}_{\|}\right)_{N}
$$

We solve Equation (18) with an Arnoldi algorithm [38] for modes with the $N_{e}$ largest eigenvalues $\left|k_{z}^{-1}\right|$ and, hence, smallest $\left|k_{z}\right|$. (To the best of our knowledge, Arnoldi is still one of the fastest iterative eigensolvers for non-Hermitian operators.) Note that the described formalism apparently yields duplicate modes $\left(\mathbf{v}_{\|}\right)_{N}$, with eigenvalues $k_{z}+G_{\perp}$ (with $G_{\perp}$ denoting all distinct linear combinations of $\mathbf{a}_{i} \cdot \mathbf{e}_{z}$ ) that are all equivalent, if $N_{e}$ is large enough. Duplicate modes therefore need to be identified (by their eigenvalue) and removed from the solution space basis to obtain a non-singular scattering problem; Section 4.

As the modes with the smallest eigenvalues of $k_{z}$ are the ones that are physically most relevant, an inversion of the Schur complement is necessary to use the Arnoldi algorithm efficiently. Therefore, at least in the straightforward implementation described here, it is necessary to store the full matrix. The computational cost (LU-factorization and matrix-vector multiplication) and the storage demand hence both scale with $N^{2}$, where the latter defines a practical hard limit for any machine: e.g., 6 GB of memory are necessary for $N=5000$ plane waves to store $S$ alone (assuming complex double entries that use $16 \mathrm{~B}$ each); while, in practice, including the factorization is 3-4-times as much.

Any similar algorithm solving Equation (18) is therefore limited to very small discretization sizes. However, the exact spatial representation for each of the plane wave components and flexibility in the cut-off enable us to obtain reasonable convergence (below one percent for $N \approx 1000$ ), as demonstrated in the next section.

Well-established plane wave methods (like MPB) that compute the ordinary band structure $k_{0}(\mathbf{k})$ for a real valued wave vector and a dielectric $\mathrm{PhC}$ do not suffer from the same intricacy, as direct matrix inversion can be avoided and the operator (in the magnetic wave equation) is Hermitian. Therefore, a fast Fourier transform in combination with the diagonal material matrices in real space and derivative matrices in reciprocal space can be used that has a smaller computational cost proportional to $N \log (N)$ and, more importantly, memory usage that only scales with $N$ [17].

The approach described in Section 3.1 has two important advantages compared to discrete Fourier transformations for a small number of plane waves $N$ that include:

(1) Precise geometrical representation: The Fourier coefficients for the material constants are calculated to machine precision compared to $N^{-1}$ precision for the fast Fourier transformation [35].

(2) Flexible cut-off: We use a spherical cut-off, including the plane waves corresponding to the $N$ smallest $\mathbf{G}$ vectors. This choice is neither isotropic nor unique for most $N$. However, it is motivated by the trivial solution for constant material functions, where the magnitude of $k_{z}$ is 
directly correlated to the magnitude of $G$. Large $G$ plane wave contributions scale in particular in first order perturbation theory with $1 /|\mathbf{G}|$. In that sense, the spherical cut-off is better suited than the three-dimensional fast Fourier cut-off, which is always rectangular [39]. This helps to use a fixed small $N$ more efficiently. In order to discretize the ball with radius $R_{N}$ in reciprocal space that contains $N$ plane waves, the fast Fourier method requires the evaluation of modes inside a parallelepiped encompassing this ball. Modes that are in the parallelepiped, but not in the ball, do not enhance the precision, but slow down computations. For the simple, face-centered and body-centered cubic lattice systems 1.9, 3.8 and 7.6, as many points are thus necessary to obtain the same precision in the limit $N \rightarrow \infty$. For finite $N$, the ratios are even larger. As an example, consider 1000 plane waves in the spherical cut-off. The number of plane waves that need to be taken into account for the parallelepiped cut-off to obtain the same precision is at least 2197, 4096 and 8000 for the three cubic lattice systems.

We show in Section 5 that the above advantages lead to substantially improved accuracy of the eigenvalues for small $N \lesssim 5000$.

\section{Scattering Problem}

In this section, we solve the scattering problem where a semi-infinite or a finite slab of $\mathrm{PhC}$ material is illuminated by a plane wave, using the solutions of Equation (11). We first construct the correct solution from the countably infinite number of modes in all domains in Section 4.1 and then describe the procedure to numerically calculate the reflection and transmission rates in Section 4.2.

\subsection{Exact Solution}

We look for the unique solution of Equation (5) for an empty half-space $\mathbb{R}^{2} \times(-\infty, 0)$ (referred to as the air domain with $\varepsilon=\mu=\mathbb{1}$ and $\zeta=0)$ and the other half $\mathbb{R}^{2} \times(0, \infty)$ given by the PhC structure with one plane wave source at $z=-\infty$ and no external sources elsewhere, similar to the situation illustrated in Figure 2. A general solution in the air domain is given by any superposition of all plane waves:

$$
\mathbf{F}=\mathbf{F}_{0} e^{\imath \mathbf{k} \cdot \mathbf{r}}
$$

that solve Equation (5), i.e., if $\mathbf{k}^{2}=k_{0}^{2}$ and $\mathbf{H}_{0}, \mathbf{E}_{0}$ and $\mathbf{k}$ form an orthogonal trihedron $\left(\mathbf{H}_{0} \cdot \mathbf{E}_{0}=\mathbf{H}_{0} \cdot \mathbf{k}=\mathbf{E}_{0} \cdot \mathbf{k}=0\right)$. Let the plane wave source emit monochromatic light with a fixed frequency $\omega=c k_{0}$ at a polar angle $\vartheta$ and an azimuthal angle $\varphi$, so that the wave vector of the incoming light is fixed by $\mathbf{k}=k_{0}(\sin \vartheta \cos \varphi, \sin \vartheta \sin \varphi, \cos \vartheta)^{T}$ and with $\mathbf{E}_{0} \cdot \mathbf{e}_{z}=0$ (s polarized) or $\mathbf{H}_{0} \cdot \mathbf{e}_{z}=0$ ( $p$ polarized), respectively. (For normal incidence, these polarization states are evidently not well defined. The two states with $\mathbf{E}_{0} \cdot \mathbf{e}_{y}=0$ and $\mathbf{H}_{0} \cdot \mathbf{e}_{y}=0$ can be used instead.) The geometry is laterally periodic with $f\left(\mathbf{r}+\mathbf{T}_{\|}\right)=f(\mathbf{r})$ ( $\mathbf{T}_{\|}$being any linear combination of $\mathbf{a}_{\|}^{(i)}=\mathbf{a}_{i}-\mathbf{e}_{z}\left(\mathbf{e}_{z} \cdot \mathbf{a}_{i}\right)$ ) for any point $\mathbf{r} \in \mathbb{R}^{3}$. It is evident that the vectors $\mathbf{a}_{\|}^{(i)}$ are only finite and can be obtained by the projection given above if the inclination direction $[h k l]$ described by the vector $h \mathbf{a}_{1}+k \mathbf{a}_{2}+l \mathbf{a}_{3}$ has Miller indices that are finite $h, k, l<\infty$. This is no practical restriction, since inclination at high symmetry directions with small $h, k, l$ are of particular interest, and all directions $\mathbf{e}_{z}$ described by finite $[h k l]$ still form a set that is dense on the unit sphere. 
The complete solution in the whole space $\mathbb{R}^{3}$ is hence laterally Bloch-periodic ( $c f$. Section 2.3) with the wave vector fixed by the plane wave source above:

$$
\mathbf{F}=\mathbf{F}(z) P(x, y) e^{\imath k_{0} \sin \vartheta(x \cos \varphi+y \sin \varphi)}
$$

Equation (20) selects all possible states for the actual scattering problem from the general solution for the left half space provided by Equation (19):

$$
\mathbf{F}_{\sigma, \mathbf{G}_{\|}}^{( \pm)}(\mathbf{r})=\mathbf{F}_{\sigma}\left(q_{ \pm}\right) e^{\imath\left(\mathbf{k}_{\|}+\mathbf{G}_{\|}+q_{ \pm} \mathbf{e}_{z}\right) \cdot \mathbf{r}}
$$

where $\sigma \in\{s, p\}$ denotes the polarization state and the $z$ component of the wave vector is determined by the dispersion relation $q_{ \pm}= \pm \sqrt{k_{0}^{2}-\left(\mathbf{k}_{\|}+\mathbf{G}_{\|}\right)^{2}}$. Note that a similar result is well known in grating theory and was derived, amongst others, by Lord Rayleigh [40]. The general solution is a linear combination of those countably infinite basis states:

$$
\mathbf{F}_{\text {air }}(\mathbf{r})=\mathbf{F}_{\sigma_{0}, 0}^{(+)}(\mathbf{r})+\sum_{\sigma, \mathbf{G}_{\|}} c_{\sigma, \mathbf{G}_{\|}} \mathbf{F}_{\sigma, \mathbf{G}_{\|}}^{(-)}(\mathbf{r})
$$

where $\sigma_{0}$ denotes the polarization state of the plane wave source with amplitude one. All remaining $\mathbf{F}_{\sigma, \mathbf{G}}^{(+)}$ have zero coefficients due to either a finite energy constraint at $z=-\infty$ (for a purely imaginary square root in Equation (21)) or the absence of a driving source for fields with energy transfer in the positive $z$-direction.

Equation (11) for the right half space yielding solutions $\mathbf{v}_{\lambda}(\mathbf{r})$ with corresponding eigenvalue $k_{z}^{(\lambda)}$ $(\lambda \in \mathbb{N})$ forms a complete solution basis in the $\mathrm{PhC}$ domain that is conformal with the lateral periodicity. A general solution for the semi-infinite scattering problem is thus given by:

$$
\mathbf{F}_{\mathrm{PhC}}(\mathbf{r})=\sum_{\lambda \in \tilde{\mathbb{N}}} c_{\lambda} \mathbf{v}_{\lambda}(\mathbf{r}) e^{\imath \mathbf{k}_{\lambda} \cdot \mathbf{r}}
$$

where $\tilde{\mathbb{N}}$ denotes the subset of $\mathbb{N}$ for which the mode corresponding to $\lambda \in \tilde{\mathbb{N}}$ is either evanescent towards positive $z\left(\operatorname{Im}\left\{k_{z}^{(\lambda)}\right\}>0\right)$ or with an average energy transfer to positive $z$ if purely propagating $\left(\operatorname{Im}\left\{k_{z}^{(\lambda)}\right\}=0\right)$. The sign of the average energy transfer is measured by the sign of the time-averaged Poynting vector integrated over one unit cell:

$$
\left\langle S_{z}^{(\lambda)}\right\rangle_{\Omega}=\frac{k_{0}}{4}\left(\mathbf{v}_{\|}^{(\lambda)}, M_{\|} \mathbf{v}_{\|}^{(\lambda)}\right)
$$

The Maxwell boundary conditions at the interface are:

$$
\mathbf{F}_{\text {air }}^{(\|)}(x, y, z=0)=\mathbf{F}_{\mathrm{PhC}}^{(\|)}\left(x, y, z=z_{0}\right)
$$

The $\mathrm{PhC}$ termination at $z_{0}$ yields an infinite set of non-homogeneous equations:

$$
\sum_{\sigma, \mathbf{G}_{\|}^{\prime}} \mathbf{F}_{\sigma, \|}\left(q_{-}\right) \delta_{\mathbf{G}_{\|}, \mathbf{G}_{\|}^{\prime}} r_{\sigma, \mathbf{G}_{\|}^{\prime}}+\sum_{\lambda \in \mathbb{N}_{+}}\left(\sum_{\mathbf{G}_{\perp}} \mathbf{v}_{\mathbf{G}, \|}^{(\lambda)} e^{\imath\left(k_{z}+G_{\perp}\right) z_{0}}\right) t_{\lambda}=\mathbf{F}_{\sigma_{0}, \|}\left(q_{+}\right) \delta_{\mathbf{G}, 0}
$$

Maxwell's Equation (5) is therefore solved in both half-spaces and at the boundary if the mode coefficients are such that Equation (25) is satisfied for any Bragg order. 
Scattering at a finite slab is done in the same way, except that in that case, the Bloch mode sum iterates over all $\lambda \in \mathbb{N}$, and there is a second set of homogeneous equations similar to Equation (25) for the right interface that involves the transmission amplitudes $t_{\sigma, \mathbf{G}_{\|}^{\prime}}$, whereas the Bloch mode amplitudes play the role of transfer amplitudes $\tau_{\lambda}$ in both sets of equations.

\subsection{Algebraic Solution with a Numerical Cut-off}

Equation (25) can be solved for both $\sigma_{0}$ numerically for the finite number of Bragg orders $N_{b}$ with the smallest $\left|\mathbf{G}_{\|}\right|$and using the $2 N_{b}$ linear independent Bloch modes with the smallest $\left|k_{z}\right|$ to obtain the $2 N_{b}$ reflection amplitudes $r_{\sigma, \mathbf{G}_{\|}^{\prime}}$ and the $2 N_{b}$ transmission amplitudes $t_{\lambda}$. (For the same reason given for the Fourier cut-off in Section 3.2, the absolute value of $k_{z}$ is correlated to the Bragg order and justifies the chosen cut-offs.) As the scattering part is, in terms of computational cost, negligible compared to the calculation of the $\mathrm{PhC}$ eigenmodes, we here calculate the full scattering matrix that handles incoming plane waves in different Bragg orders (if existing) and from both sides of a finite slab.

The interfacial scattering matrices are obtained in the following, where the input port is referred to as left and the output as right. The modes on the left of the interface are labelled by 1 and the modes on the right by 2 . Further, the modes that are propagating or increase their energy towards the right or left are labelled + or - , respectively. The four types of mode coefficient vectors of length $2 N_{b}$ are then given by $\mathbf{c}_{(1,2)}^{( \pm)}$, and the corresponding scattering matrix is:

$$
\left(\begin{array}{l}
c_{1}^{(-)} \\
c_{2}^{(+)}
\end{array}\right)=\left(\begin{array}{ll}
S_{11} & S_{12} \\
S_{21} & S_{22}
\end{array}\right)\left(\begin{array}{l}
c_{1}^{(+)} \\
c_{2}^{(-)}
\end{array}\right)
$$

The four matrix sub-blocks can be interpreted as left reflection $S_{11}$, right reflection $S_{22}$, transmission from left to right $S_{21}$ and transmission from right to left $S_{12}$ ( $c f$. Figure 4 in [12]).

These coefficient vectors are multiplied from the left by the field matrices to satisfy the Maxwell boundary conditions; Equation (24). For each Bragg order $i$, we define the orthonormal planar basis $\mathbf{e}_{1}^{(i)}=\left(\mathbf{k}_{\|}+\mathbf{G}_{\|}\right) /\left|\mathbf{k}_{\|}+\mathbf{G}_{\|}\right|$and $\mathbf{e}_{2}^{(i)}=\mathbf{n}_{\text {int }} \times \mathbf{e}_{1}$ with the surface normal $\mathbf{n}_{\text {int }}$ of the interface between air and $\mathrm{PhC}$. Using this basis, the entries of the vacuum field matrices can be expressed by:

$$
F_{i j}^{( \pm)}=\delta_{i j} F_{i}^{( \pm)}
$$

where $i, j$ denote the different Bragg orders, and the $4 \times 2$ field matrix for each Bragg order:

$$
F_{i}^{( \pm)}:=\left(\begin{array}{ll}
H_{s 1}^{( \pm)} & H_{p 1}^{( \pm)} \\
H_{s 2}^{( \pm)} & H_{p 2}^{( \pm)} \\
E_{s 1}^{( \pm)} & E_{p 1}^{( \pm)} \\
E_{s 2}^{( \pm)} & E_{p 2}^{( \pm)}
\end{array}\right)=\left(\begin{array}{cc}
-q_{ \pm} & 0 \\
0 & k_{0} \\
0 & q_{ \pm} \\
k_{0} & 0
\end{array}\right)
$$

is normalized, so that the time-averaged Poynting vector in the $z$-direction is $S_{z}=\left(k_{0} / 2\right) \operatorname{Re}\left\{q_{ \pm}\right\}$ for propagating modes. The $\mathrm{PhC}$ field matrix at the air/ $\mathrm{PhC}$ interface is evaluated at the termination value $z_{0}$ :

$$
V_{i \lambda}^{( \pm)}\left(z_{0}\right)=\sum_{G_{\perp}} T_{i}\left(\mathbf{v}_{\|}\right)_{i \lambda}^{( \pm)} e^{\imath\left(G_{\perp}+k_{z}\right) z_{0}}
$$


where $z_{0}$ is the position relative to the origin choice in Equation (18), and $T_{i}$ converts from the coordinate system used in Equation (18) to the Bragg order basis, i.e., $T_{i}=\left[\mathbf{e}_{1}^{(i)}, \mathbf{e}_{2}^{(i)}\right]^{T}$. The scattering matrices for the left and right interfaces are thus given by:

$$
S_{l}=\left(F^{(-)}, V^{(+)}\left(z_{l}\right)\right)^{-1}\left(F^{(+)}, V^{(-)}\left(z_{l}\right)\right) \quad \text { and } \quad S_{r}=\left(V^{(-)}\left(z_{r}\right), F^{(+)}\right)^{-1}\left(V^{(+)}\left(z_{r}\right), F^{(-)}\right)
$$

where $z_{l}$ and $z_{r}$ determine both the crystal terminations and the thickness $d=z_{r}-z_{l}$ of the structure and should for reasons of numerical stability [11] be chosen, such that $z_{l} \leqslant 0$ and $z_{r}>0$, which is always possible if $d>a$. (The case $d<a$ does not yield useful physical features that are not also obtained by a simpler two-dimensional grating structure, as well, nor are the eigenmodes of an infinitely periodic structure (from which a part is not present in the actual scattering geometry) a good basis choice in which to express the fields within the slab.)

For a semi-infinite slab, the reflection and transmission amplitude vectors are obtained by multiplication of the left interface scattering matrix with the coefficient vector of the incoming plane wave, i.e., the vector with all of the $4 N_{b}$ entries vanishing, except for the zero Bragg order in $\mathbf{c}_{1}^{(+)}$:

$$
\left(\begin{array}{l}
r \\
t
\end{array}\right)=\left(\begin{array}{c}
S_{11}^{(l)} \\
S_{21}^{(l)}
\end{array}\right) \mathbf{c}_{1}^{(+)}
$$

The scattering matrix for a finite slab is obtained by the Redheffer star product (Equation (22) in [12]) between both interfacial scattering matrices (no phase matrix as in the generalized Airy formulae [41] are necessary due to the global choice of origin for $S_{l}$ and $S_{r}$.):

$$
\begin{aligned}
& S_{11}=S_{11}^{(l)}+S_{12}^{(l)} S_{11}^{(r)}\left[\mathbb{1}-S_{22}^{(l)} S_{11}^{(r)}\right]^{-1} S_{21}^{(l)} \\
& S_{21}=S_{21}^{(r)}\left[\mathbb{1}-S_{22}^{(l)} S_{11}^{(r)}\right]^{-1} S_{21}^{(l)}
\end{aligned}
$$

and two similar equations (which are irrelevant if light is only incident from one side of the PhC slab) with the index exchanges $1 \leftrightarrow 2$ and $l \leftrightarrow r$. Note that the matrix inverse represents a Neumann series that covers all contributions from wave packages that are arbitrarily often passing through the structure via successive internal reflection at the right interface followed by internal reflection at the left interface.

As for the semi-infinite slab, reflection and transmission amplitude vectors are given by the product of the scattering matrix with the coefficient vector of the incoming plane wave:

$$
\left(\begin{array}{l}
r \\
t
\end{array}\right)=\left(\begin{array}{l}
S_{11} \\
S_{21}
\end{array}\right) \mathbf{c}_{1}^{(+)}
$$

\section{Specific PhC Geometries}

The convergence characteristics of our method are demonstrated by two numerical examples: a Bragg mirror for which the analytical solution is known, and an inverse (complement) simple cubic sphere packing. The convergence rate is generally only linear, limited by the Gibbs phenomenon. However, coupling to an incident plane wave, which is of theoretical interest and naturally performed in the Fourier basis projected onto the surface, suffers from the same phenomenon, even if the eigenstates are exactly known as for a slice in the lamellar grating [10]. 
As an application, we also calculate the complex band structure of a non-chiral simple cubic truncated octahedron ( $c f$. Figure 4) made of a material with intrinsic chirality and the chiral body-centered cubic gyroid structure (see, for example, $[5,15]$ ). The results are analysed in detail for both geometries.

\subsection{Convergence Behaviour}

The convergence behaviour is quantified by the absolute value of the relative difference between the eigenvalue for a discretization of $N$ plane waves $k_{z}^{(N)}$ and the analytical solution $k_{z}^{(\infty)}$, i.e., $\delta k_{z}(N)=\left|1-k_{z}^{(N)} / k_{z}^{(\infty)}\right|$. The convergence rate is said to be of order $o$ if $\lim _{N \rightarrow \infty} \delta k_{z}(N) \propto N^{-o / d}$ (note the factor $1 / d$ in the exponent that stems from the spatial dimension $d$ of the problem); it is called linear, quadratic and cubic if $o=1,2,3$, respectively.

A one-dimensional PhC with a unit cell $\Omega=\mathbb{R}^{2} \times(0, a)$ and the two material domains $\Omega_{0}=\mathbb{R}^{2} \times$ $\left(d_{1} / 2, a-d_{1} / 2\right)$ and $\Omega_{1}=\Omega \backslash \Omega_{0}$ is referred to as a Bragg mirror [2]. The analytical solution can be found either by separation of variables [10] of the Bloch mode equation or by interface matching of the single domain solutions using the Bloch boundary condition [42]. There are four solutions for a given angle of incidence and frequency with the eigenvalue of the form $k_{z}^{( \pm, \pm)}= \pm \arccos \left(A \pm \sqrt{A^{2}-B}\right)$, where both $A$ and $B$ are functions of the frequency, the parallel wave vector and the structure parameters $\left(d_{i}, \varepsilon_{i}, \mu_{i}, \zeta_{i}\right)[42]$.

We use the analytical solution for $k_{z}^{(\infty)}$ to quantify the convergence behaviour of our method for a Bragg mirror; Figure 6. The wave number $k_{z}$ converges with cubic order in the case of normal incidence (Figure 6A) and linearly otherwise (Figure 6B). This discrepancy can be explained by the Gibbs phenomenon [16]: all field components of the eigenmodes are continuous and continuously differentiable in the first case, while the $z$ component of both the $\mathbf{E}$ and the $\mathbf{H}$ field are discontinuous in the second case, yielding, respectively, cubic and linear convergence behaviour for our method that is based on a global Fourier expansion of the fields.

We also investigate the convergence behaviour for a more realistic three-dimensional example of air spheres of radius $0.3 a$ in a dielectric matrix with $\varepsilon=12$. In this case, we compare our results with those of MPB (Figure 7), as an analytic reference solution is not available. We first calculate the eigenfrequencies for the wave vector $\mathbf{k} a /(2 \pi)=(0,0,0.2)^{T}$ using MPB with a high resolution of 128 (i.e., $N=128^{3} \approx 2 \times 10^{6}$ ) and a sub-pixel mesh of seven [17] for averaging the dielectric constant at the interfaces. The resulting frequencies for the first and the third band are then used to set $k_{0}$ for our algorithm, and the relative error of $k_{z}$ is calculated with respect to $k_{z}^{(\infty)} \approx 0.2$. We compare the relative error of our results to MPB results for the same discretization range and a relative error calculated with respect to the $N=128^{3}$ solution. 
A

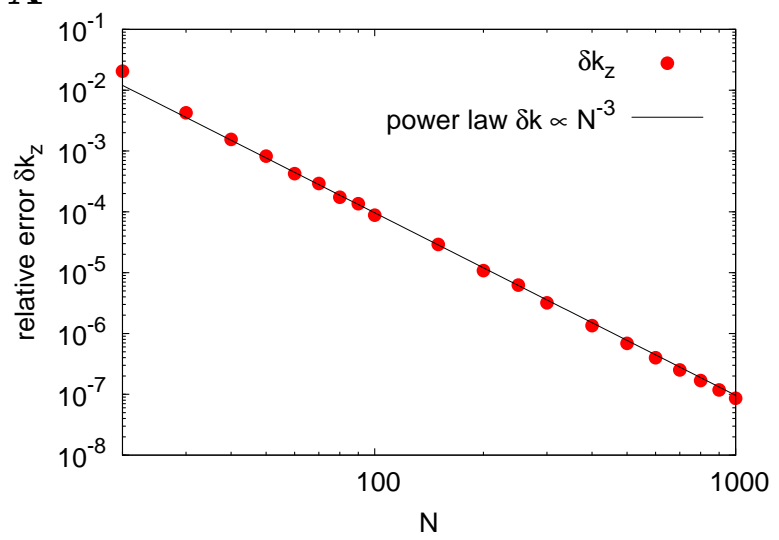

B

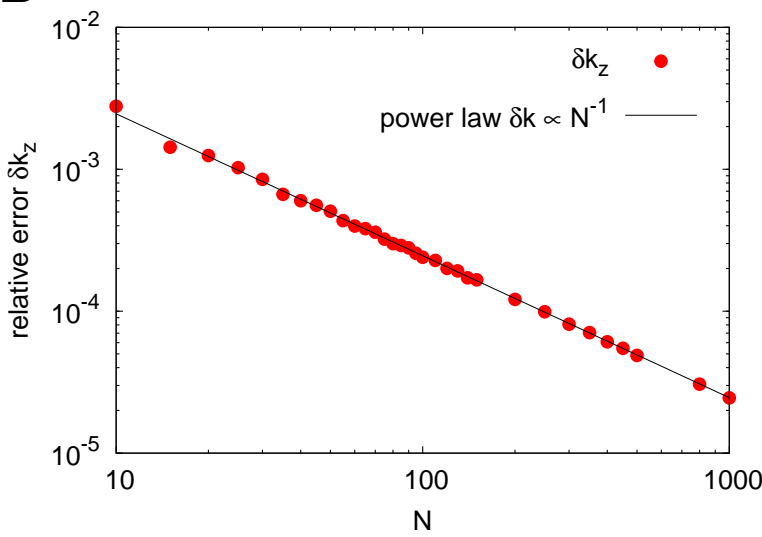

Figure 6. Convergence analysis for the smallest eigenvalue $k_{z}$ for two different Bragg mirrors coupled to normal and $25^{\circ}$ incidence. (A) Normal incidence onto a Bragg mirror made of an alternating slicing of a high index material $\left(\varepsilon_{1}=12+0.1 \imath, \mu_{1}=1\right.$, $\left.\zeta_{1}=0\right)$ and a strongly left-rotating low index material $\left(\varepsilon_{2}=2.4+0.01 j, \mu=1\right.$, $\zeta_{2}=0.3 \imath$ ) with respective thickness $d_{1}=0.7 a$ and $d_{2}=0.3 a$. The reduced frequency is set to $\omega a /(2 \pi c)=1.8$. (B) Incidence at $25^{\circ}$ onto a Bragg mirror defined by the domain parameters $d_{1}=0.3 a, \varepsilon_{1}=6+0.2 \imath, \mu_{1}=2, \zeta_{1}=0.5 \imath$ and $d_{2}=0.7 a, \varepsilon_{2}=1, \mu_{2}=1$, $\zeta_{2}=0$, i.e., an alternation of thin arbitrary artificial material and air, with $\omega a /(2 \pi c)=0.1$.

First band

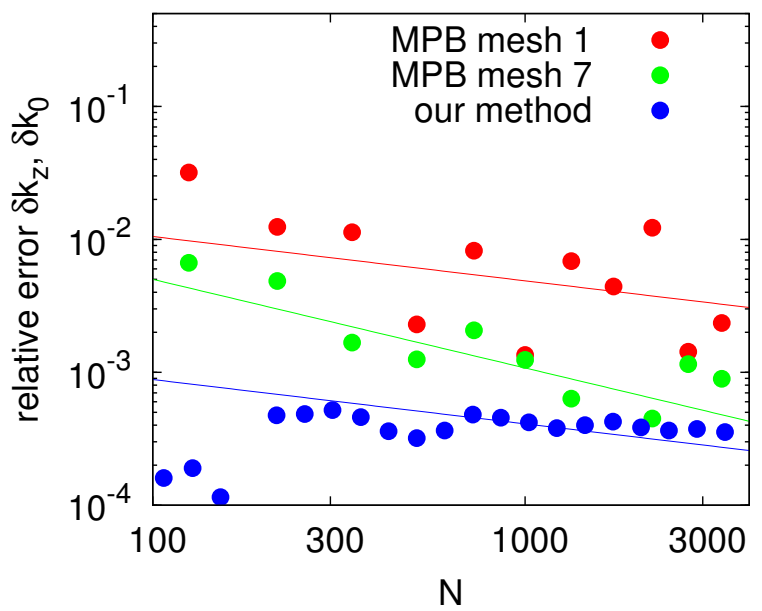

Third band

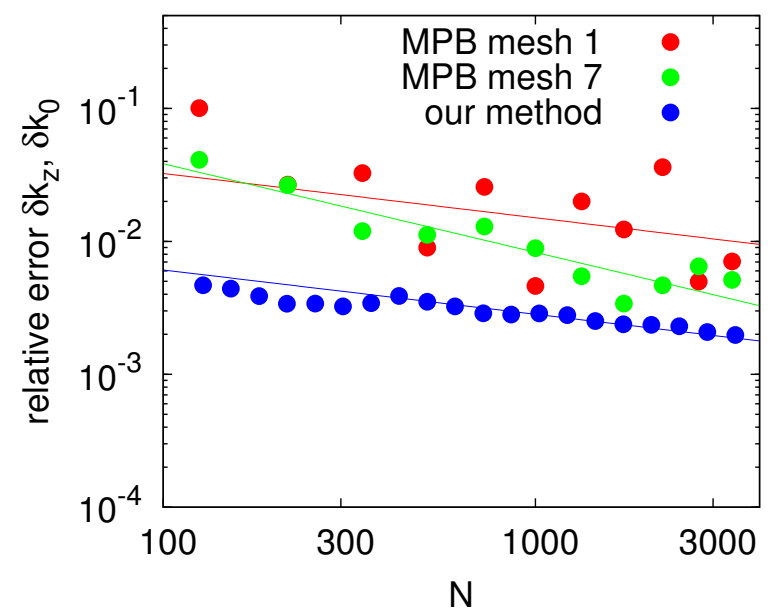

Figure 7. Convergence analysis for a simple cubic structure consisting of air spheres in a silicon matrix $(\varepsilon=12)$. The points represent the relative error of MPB and our method (with respect to a converged MPB solution $k_{0}^{(N)}\left(k_{z}=0.2\right)$ with $N=128^{3}$ and a mesh size of seven) for different numbers of plane waves up to $N \approx 4000$, above which memory requirements become unfeasible on most machines. The spatial structure is represented by a triangulated sphere with 384 triangles generated with Ken Brakke's surface evolver [43] for our method and generated with the internal geometry class sphere in the case of MPB. Straight lines represent power law fits $\delta k_{z}, \delta k_{0} \propto N^{-1 / 3}$ for our method and MPB without $\varepsilon$-averaging (mesh 1) and $\delta k_{0} \propto N^{-2 / 3}$ with averaging (on a mesh of $7^{3}$ sub-pixels) [17]. 
Note that this comparison serves solely the purpose to show that the triangulated exact Fourier transformation is superior to the fast Fourier transformation if the eigenvalues $k_{z}\left(k_{0}, \mathbf{k}_{\|}\right)$, rather than $k_{0}(\mathbf{k})$, are needed, and hence, a full matrix representation of the material constants is necessary. If this is not the case, the standard procedure [17] without full matrix storage is much better suited and allows system sizes that are $\approx 3$ orders of magnitude larger. Figure 7 shows that the numerical error of our method is below $1 \%$, even for very small discretization sizes $N \approx 100$ and below the results obtained with MPB in the range of interest. The expected convergence rates, linear in our case and for MPB without averaging, quadratic for MPB with averaging, fit the data well. The coefficient largely depends on the quality of geometrical representation that is approximated with linear (quadratic) accuracy in the case of MPB and with negligible error in our case and on the field intensities of the respective mode near the surface where the plane wave expansion produces a large deviation (linear rate without averaging) from the true solution.

\subsection{Truncated Octahedron}

As a three-dimensional test case with a non-chiral geometry, we use a simple cubic crystal that is generated from a dual lattice of truncated octahedra ( $c f$. Figure 4), with the interface closely related to the Kelvin foam [44]. This geometry is of $P m \overline{3} m$ symmetry (as in a $\mathrm{CsCl}$ crystal) and is topologically equivalent to the triply-periodic minimal surface structure [45] in which the two spatial domains are separated by the oriented Schwarz Psurface [46].

In the actual $\mathrm{PhC}$ under investigation, one of the two domains of this structure is left empty (air) and the other made of an artificial material that we call rotion (The name is motivated by the property that the semi-major axisof the polarization ellipse of a plane wave travelling through the bulk material is rotated due to finite coupling constant $\zeta$. We introduce rotion with the frequency independent coupling constant only to demonstrate the validity of our algorithm. For a physical material, the rotation length is expected to be in first order relative to the wave length and, hence, $\zeta \propto \omega \omega[29]$.) with permittivity $\varepsilon=12+0.1 \imath$ close to silicon, permeability $\mu=1.5$ and intrinsic chirality corresponding to $\zeta=0.2 \imath$. For this $\mathrm{PhC}$, Figure 8 shows the reduced frequency plotted over the real part (the propagation constant $k$, left) and the imaginary part (the decay rate $\kappa$, right) of the reduced wave number in the [100] surface inclination direction. This kind of plot is referred to as a complex band structure (CBS) diagram in the following.

In the band structure plots shown here, the different colors and point sizes (referred to as the mode profile) represent, respectively, the circular polarization index and the coupling index. The color bar ranges from blue (LCP-philic, where LCP is the abbreviation of left circularly polarized) over black (no preferred polarization) to red (RCP-philic, where RCP is the abbreviation of right circularly polarized); modes with small points show weak coupling to a plane wave compared to those with a larger point size. A more formal definition is given in [5,18]; the actual color and size code is the same as is in Figure 4 in [18].

While the mode profile clearly indicates that the material corresponding to Figure 8 is indeed chiral, the color scheme mainly serves here as a guide to the eye to identify corresponding points on the left and right part of the CBS diagrams. As both diagrams can only be read simultaneously, we do not show the 
propagation constant of a mode whose decay rate is outside of the range of the right ordinate and vice versa; see, for example, the two bands that seem to end at $\star$ in Figure 8.
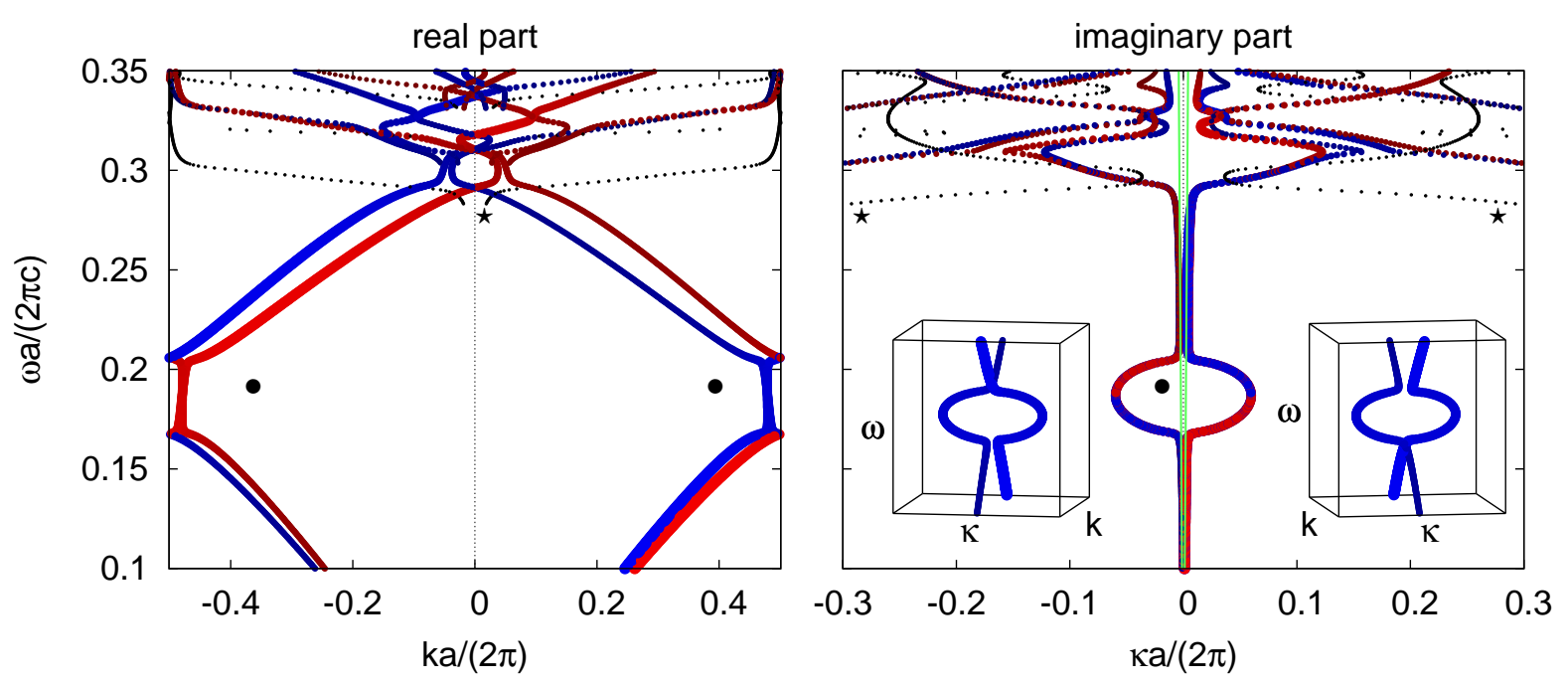

Figure 8. Complex band structure along the [100] direction of the truncated octahedron photonic crystal with intrinsic material chirality. The parallel wave vector is set to zero (corresponding to normal incidence); the Galerkin space contains $N=2000$ plane waves.

None of the Bloch modes are purely propagating with $\kappa=0$ caused by the finite energy dissipation within the rotion material region of the $\mathrm{PhC}$. The green lines in Figure 8 represent the modes in a homogeneous slab of rotion, where $k_{0}= \pm\left(2 n_{ \pm} / \operatorname{Im}\{\varepsilon\}\right) \kappa$ with the refractive index $n_{ \pm}=\sqrt{\operatorname{Re}\{\varepsilon\} \operatorname{Re}\{\mu\}} \pm \zeta$ is a good approximation [31]. The decay rate for all $\mathrm{PhC}$ modes is stronger, except for the low frequency limit, where the $\mathrm{PhC}$ can be approximated by a homogeneous effective medium [47] with material constants between those of rotion and air. (A Bragg mirror structure yields $f_{\text {eff }}^{(z z)}=\sum_{i} \varphi_{i} f_{i}$ and $f_{\text {eff }}^{(x x)}=f_{\text {eff }}^{(y y)}=\tilde{f} /\left(\tilde{\mu} \tilde{\varepsilon}+\tilde{\zeta}^{2}\right)$ with $\tilde{f}=\sum_{i} \varphi_{i} f_{i} /\left(\mu_{i} \varepsilon_{i}+\zeta_{i}^{2}\right)$. Aspnes argues for the special case of $\mu=1$ and $\zeta=0$, that any effective constant must have a value between those two extrema that are contributions of interface parts parallel and perpendicular to a field component. Evidently, an effective constant for a three-dimensional $\mathrm{PhC}$ cannot be described by a tensor and must instead be described by a function of the solid angle of $\mathbf{k}$.)

The insets in the right half of Figure 8 show a three-dimensional plot of the LCP-philic modes in the box of dimensions $0.3<k a /(2 \pi)<0.7,-0.1<\kappa a /(2 \pi)<0.1$ and $0.12<\omega a /(2 \pi c)<0.25$ from two different angles. These insets illustrate that due to finite absorption, 2 LCP-philic single bands that are at no point degenerate exist over the whole frequency range (an explanation is given in Section 5.3). For the RCP-philic case, the situation is similar, although the actual position of the bands compared to the LCP case is perturbed on the $k$ axis. This feature cannot be seen in the regular CBS diagram, as especially in the bandgap (•), two LCP and two RCP bands are $k$-degenerate, while, respectively, one LCP and one RCP band are $\kappa$-degenerate.

The CBS diagram without the mode profile for the same $\mathrm{PhC}$ is shown again in Figure 9 for three different numbers of plane waves $N=500,1500$ and 2000, to demonstrate the well-behaved convergence of the solutions of Equation (18). The $k, \kappa$ values for $N=2000$ lie on top of those for 
$N=1500$, except for flat bands $\left(\partial k_{0} / \partial k \ll 1\right.$ or $\left.\partial k_{0} / \partial \kappa \ll 1\right)$, where the solution appears far from converged as a result of the frequency discretization.
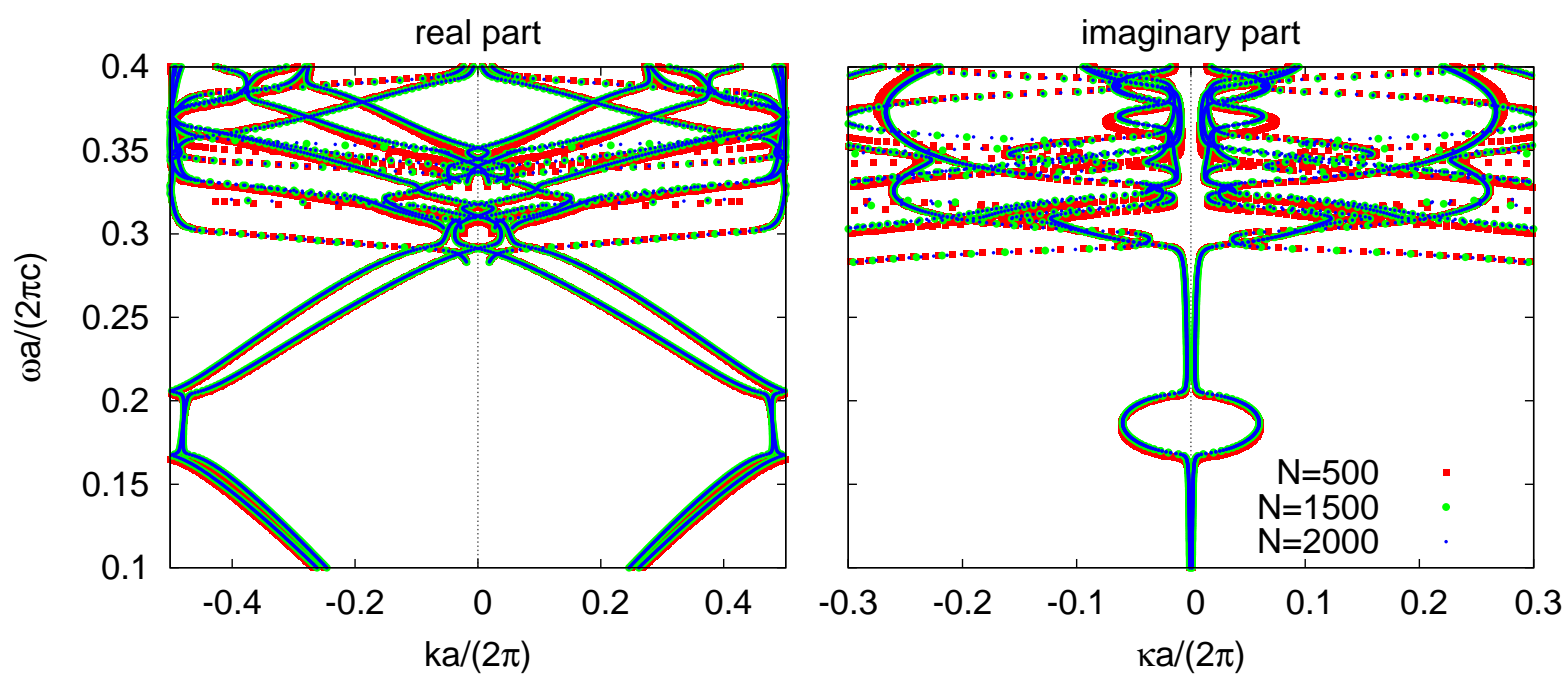

Figure 9. Well-behaved convergence for the photonic crystal based on the Kelvin body. The graph shows the complex band structure (CBS) diagram for the same photonic crystal as discussed in Figure 9 for three different numbers of plane waves.

\subsection{Complex Band Structure of and Reflectance at the Single Gyroid PhC}

The single gyroid $\mathrm{PhC}$ is composed of two interwoven material domains, each a single connected component with a network-like topology, that are separated by a gyroid constant mean curvature surface [22]. It is chiral, i.e., it cannot be superposed with its mirror image by rotations and translations alone, with body-centered cubic symmetry $I 4_{1} 32$. The single gyroid is found in many biological and chemical systems at several length scales [48] and, in particular, in butterfly PhCs [5,14,23,49,50].

The geometry of the $\mathrm{PhC}$ that is discussed in this work is defined by an interfacial surface $S$ of a mesh of $2 \times 10^{4}$ triangles based on a nodal surface approximation [45] of the constant mean curvature surface given by:

$$
t=\sin G_{0} x \cdot \cos G_{0} y+\sin G_{0} y \cdot \cos G_{0} z+\sin G_{0} z \cdot \cos G_{0} x
$$

with $G_{0}:=2 \pi / a$. The nodal surface parameter is set to $t=0.9$, so that one of the spatial domains has a volume fraction $\varphi=20.2 \%$. This domain is filled with a dielectric material of permittivity $\varepsilon=2.4$, whereas the other is left empty $(\varepsilon=1)$, with $\mu=1$ and $\zeta=0$ in both domains.

This PhC serves as model for the chiral nanostructure found in the wing scales of the butterfly, Callophrys rubi $[5,14,23,49,50]$; see Figure 1. It exists in two enantiomeric forms, which are mirror images of each other (note that we here analyse the opposite enantiomer to the one discussed in [14], leading to identical optical behaviour when LCP and RCP are exchanged).

\subsubsection{Complex Band Structure Along the Cubic [100] Direction}

Figure 10 shows the CBS diagram along the cubic [100] direction. Note the four evanescent Bloch modes with finite propagation constant and decay rate at the frequency marked by the symbol $\star$. The two weak coupling modes are perturbations of the eight vacuum modes (Another two modes are found 
mirrored at the $H$ point, due to time reversal symmetry. Three of the remaining four modes exhibit a fork in the decay rate diagram, the two other RCP and LCP modes marked with a $\star$ and another LCP band. The last band joins with the real band structure above $\omega a /(2 \pi c)=0.95$ and is only visible in the decay rate diagram.), with $\mathbf{G}_{\|} a /(2 \pi)=( \pm 1,0,0)^{T}$ and $(0, \pm 1,0)^{T}$, which are equivalent to the $H$ point at $\mathbf{k} a /(2 \pi)=(0,0,1)^{T}$ and for which the decay rate is determined by $\kappa=\sqrt{1-k_{0}^{2}}$, forming a half-ellipse in the $k=$ const. plane within the $k, \kappa, \omega$ parameter space. The elliptical shape is closely reflected in the decay rate part of the CBS diagram in Figure 10, while the plane seems tilted around the $(k, \omega)=0$ axis. This evanescent band segment is continued at the minimum of the purely propagating band part above. We refer to such points where an evanescent (or mainly evanescent) band segment joins with a dispersive (or mainly dispersive) segment as a fork point.

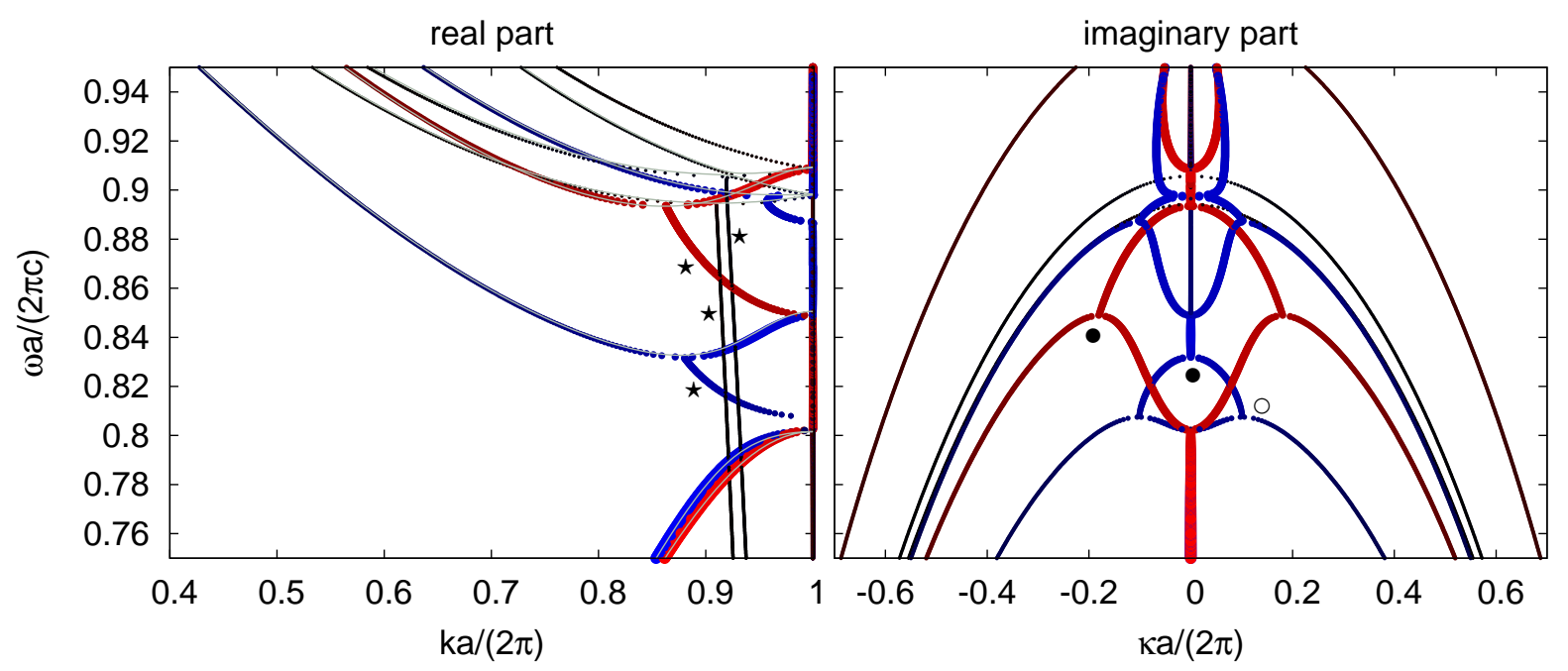

Figure 10. CBS diagram along the [100] direction of a single gyroid $\mathrm{PhC}$ calculated with $N=2007$ plane waves. The solid lines that are hardly perceivable and shown on the left represent the results of an MPB [17] calculation for $N_{\mathrm{MPB}}=64^{3}>100 \mathrm{~N}$ plane waves (and otherwise the same parameters), which serves as reference for the purely propagating Bloch modes.

The behaviour at fork points is, as shown below, a consequence of the holomorphic nature of the operator $\Theta\left(k_{z}\right)$ in Equation (5) that yields holomorphic eigenvalues $\omega_{n}\left(k_{z}\right)$ (we call them bands) [51]. At fork points $k_{z}^{(0)}$, the complex derivative $\omega^{\prime}\left(k_{z}\right)$ vanishes. As a result, the complex frequency in the vicinity of a fork point is given by its Taylor expansion:

$$
\omega\left(k_{z}\right)=\omega\left(k_{z}^{(0)}\right)+\frac{\omega^{\prime \prime}\left(k_{z}^{(0)}\right)}{2} \delta k_{z}{ }^{2}+\mathcal{O}\left(\delta k_{z}^{3}\right)
$$

with $\delta k_{z}=\left(k_{z}-k_{z}^{(0)}\right)$. As we are only interested in steady-state solutions where $\omega$ is real, the condition $\operatorname{Im}\left\{\omega^{\prime \prime} \delta k_{z}{ }^{2}\right\}=0$ applies near $k_{z}^{(0)}$ (where $\omega$ is real), i.e., $2 \operatorname{Re}\left\{\omega^{\prime \prime}\right\} \delta k \delta \kappa+\operatorname{Im}\left\{\omega^{\prime \prime}\right\}\left(\delta k^{2}-\delta \kappa^{2}\right)=0$, defining two perpendicular straight lines in the $k, \kappa$ plane. Evaluating the Taylor expansion under this constraint, one finds that the fork opens in the positive $\omega$-direction along one line and in negative direction along the other line. (if $\operatorname{Re}\left\{\omega^{\prime \prime}\right\} \neq 0$, which is the case in any physical situation with $\operatorname{Re}\{\varepsilon\}, \operatorname{Re}\{\mu\} \neq 0$ ). 
The forking behaviour can be illustrated by the complex function $\cos (z)$ with $z=x+\imath y$ and $x, y \in \mathbb{R}$. Consider the bands of this function that satisfy $\cos (z) \in \mathbb{R}$, and therefore, either $\sin (x)=0$ or $\sinh (y)=0$, describing the forked (Note that the fork points of $\Gamma$ are not to be confused with the branch points in the conventional sense of complex analysis. Specifically, the function $\cos (z)$ is single-valued and, hence, does not have branch points, but shows forking. The forking results from considering a complex function $\tilde{\omega}: \mathbb{C} \rightarrow \mathbb{C},(k, \kappa) \mapsto \tilde{\omega}(k, \kappa)$; restricted to the subset $\Gamma$ of the whole parameter space $\mathbb{C}$, where $\tilde{\omega}$ adopts real values, such that $\omega: \Gamma \subset \mathbb{C} \rightarrow \mathbb{R},(k, \kappa) \mapsto \omega(k, \kappa):=\tilde{\omega}(k, \kappa)$.) path illustrated in Figure 11 in the complex plane. The resulting CBS diagram for the real part of the function $\cos (z)$ is shown in Figure 11B.
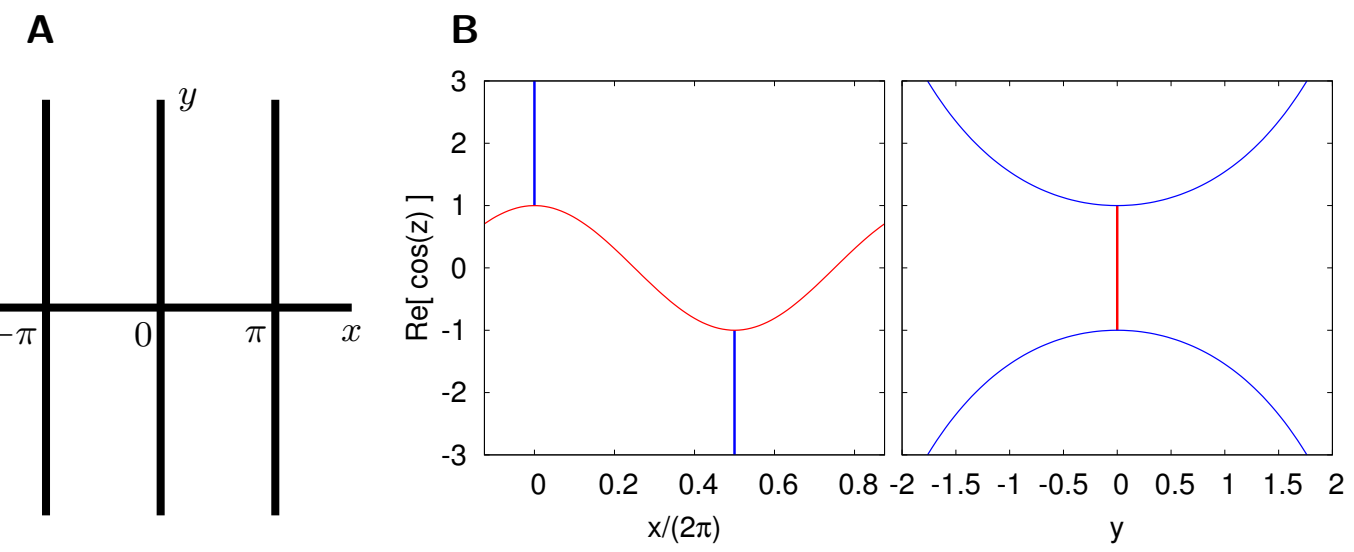

Figure 11. Illustration of the bands of the holomorphic function $\cos (z)$. (A) Schematic illustration of the forked paths, where $\operatorname{Im}\{\cos (z)\}=0$ in the imaginary plane, where $x=\operatorname{Re}\{z\}$ and $y=\operatorname{Im}\{z\} .(\mathbf{B}) \operatorname{Re}\{\cos (z)\}$ in a CBS diagram, as in Figure 8.

The evanescent parts of the bands labeled by $\star$ are joined to the propagating part via the forks that are described above. The same kind of forks also occur for non-vanishing decay rates $(\bullet)$. Note that points with $\omega^{\prime}\left(k_{z}\right)=0$ cease to exist with finite dissipation (the imaginary part is usually not zero any more). A fork undergoes a topological chance if dissipation is turned on, similar to the vertex disconnection operation in [52], and the situation for finite absorption is illustrated by the insets in the decay-rate part of Figure 8.

\subsubsection{Scattering at a Semi-Infinite Slab with [100] Inclination}

Importantly, there is a fork ( $\circ$ ) within the indirect bandgap above the fundamental bands $(0.8<\omega a /(2 \pi c)<0.83)$. The decay rate therefore increases rapidly away from the propagating band edges, compared with the ordinary oval shape of a direct bandgap in the decay rate diagram ( $c f$. Figure 8 at $\bullet$ ). This explains why the slope in the reflectivity at the same position is large for a moderate number of unit cells, as seen in Figure 12, at $\star$ for the weak saturated dashed curves that correspond to the result of an MEEP simulation calculating the average of the reflectance spectra of a normally incident plane wave that is scattered, respectively, at a finite slab with varying thickness around $6 a$ inclined in [100]. The data is taken from Figure 5A in [14]. 
The bold solid and dashed lines in Figure 12 correspond to the reflectance spectrum at a semi-infinite slab obtained by our method for $N=1503$ plane waves and $N_{b}=13$ Bragg orders and $N=2517$ plane waves and $N_{b}=21$ Bragg orders, respectively. The number of Bragg orders corresponds to a scattering Equation (25) with $2 N_{b}$ Bloch modes (including evanescent modes) and the same number of plane waves (in the air domain). All spectra are shown for RCP and LCP incident light, where we have calculated the amplitudes for $s$ and $p$ polarizations, as described in Section 4, and performed a basis change afterwards. The bold spectra contain reflectance in both polarization states (Polarization conversion where RCP incident light gets reflected as RCP light (and similar for LCP) is covered here. It is weaker than direct reflection within the frequency range shown here, yet not vanishing. This is generally the case for reflection at the single gyroid structure that has no pure $n$-fold rotation symmetry with $n>2$ in the propagation direction, the presence of which would prevent conversion, as shown in [18].) in the zero Bragg order, which is equal to the full reflectance below reduced frequency $\omega a /(2 \pi c)=1$, where all non-zero Bragg orders are evanescent in the air domain.

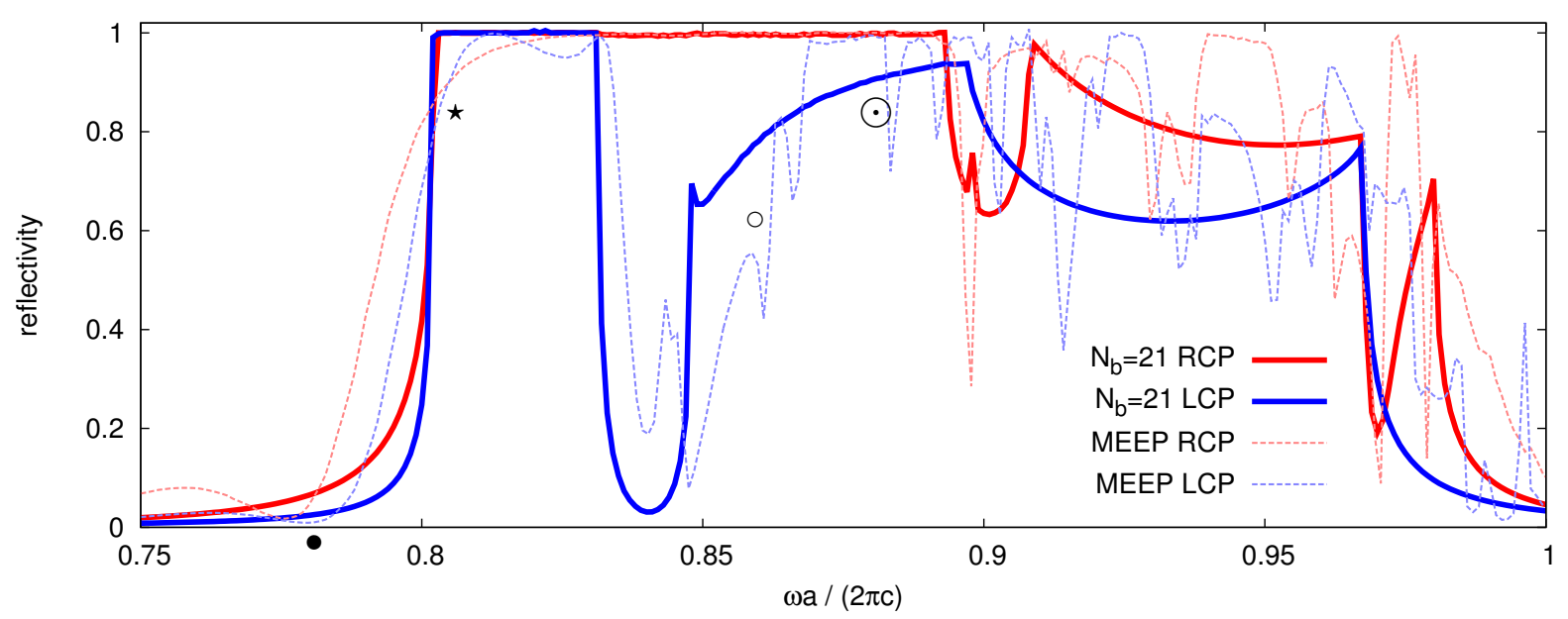

Figure 12. Reflectance spectrum for a plane wave at normal incidence onto a [100] inclined single gyroid photonic crystal ( $\mathrm{PhC}$ ), for left- and right-circularly polarized light. The parameters are the same as in Figure 10. Spectra with right circularly polarized (RCP) incident light are shown in red and with left circularly polarized (LCP) light in blue. The solid lines represent a calculation with $N=2517$ plane waves and $N_{b}=21$ Bragg orders, i.e., the $2 N_{b}=42$ Bloch modes with the smallest absolute wave number and propagating (decaying) away from the surface have been used. A computation with a smaller resolution $N=1503$ and $N_{b}=13$ shows no difference, except for the small peak at $\omega a /(2 \pi c)=0.9$, which is not resolved. The dashed lines in less saturated colors correspond to an average over a few slabs with varying thickness around $6 a$, simulated with MEEP (data from Figure 5A in [14]).

Evidently, the reflectance at the semi-infinite structure is one for frequencies where no purely propagating Bloch mode exists and no resonances of thin-film Fabry-Pérot $(\bullet)$ and Fano (e.g., at $\circ, \odot$ ) shape, which are found in finite slabs only, in the frequency region below the first wood anomaly $(\omega a /(2 \pi c)=1)$ that is shown here. 
There is an instructive explanation for the sharp resonances of Fano shape given in [41] for a two-dimensional grating slab that is homogeneous in the propagation direction. The main idea can be adopted for non-homogeneous three-dimensional PhC slabs:

The Neumann series in the Redheffer star product (Equation (32)) diverges if at a complex frequency $\omega_{0}+\imath \gamma$, the product of the internal reflection matrices $S_{22}^{(l)} S_{11}^{(r)}$ has an eigenvalue of one (all eigenvalues have a norm less than or equal to one, due to energy conservation, so that the series converges in any other case). A Fano resonance at frequency $\omega_{0}$ is close to the complex eigenfrequency $\omega_{0}+\imath \gamma$ of a slab mode, aka guided mode, determined by $\operatorname{det}\left(\mathbb{1}-S_{22}^{(l)} S_{11}^{(r)}\right)(\omega)=0$, with a high-quality factor corresponding to $\gamma \ll \omega_{0}$. A resonance with a symmetric $(\odot)$ or asymmetric (o) Fano line shape results in the reflection and transmission spectra close to $\omega_{0}$. The same type of internal matrix singularity-induced resonance does not occur for non-diffracting structures, where no guided modes can be excited, as the internal reflections are described by scalars with magnitude smaller than one below the critical angle.

Slowly modulating thin film Fabry-Pérot resonances, on the contrary, also appear in homogeneous thin-films at all angles of incidence. In the case of a $\mathrm{PhC}$, if the Fourier coefficients of all significantly involved Bloch modes only slowly modulate with frequency and if only one propagating Bloch mode acts as a transfer channel in each direction, the frequency dependence of the change of the fields due to a pass back and forth through the structure is dominated by the optical phase shift of the transfer modes $\exp \left\{\imath\left(k_{z}^{(+)}+k_{z}^{(-)}\right) d\right\}$. A sinusoidal modulation (compressed or stretched, depending on the slope of the bands) of the scattering parameters with frequency results.

Absorption evidently weakens the resonance effects described above ( $c f$. Figure 7B in [14]), as it decreases the induced norm of both $S_{22}^{(l)}$ and $S_{11}^{(r)}$ and, hence, increases the speed of convergence of the corresponding Neumann series.

\subsubsection{Complex Band Structure along the Cubic [111] Direction}

Figure 13 shows the CBS diagram of the gyroid $\mathrm{PhC}$ along its cubic [111] direction. Note that the band structure is extended beyond the Brillouin zone edge at the $\mathrm{P}$ point $\left(\mathrm{k}=(0.5,0.5,0.5)^{T} 2 \pi / a\right)$ to the $\mathrm{H}\left(\mathbf{k}=(1,1,1)^{T} 2 \pi / a \equiv(1,0,0)^{T} 2 \pi / a\right)$ point to cover all modes that are by their lateral dispersion able to couple to a wave at normal incidence.

No partial bandgap is found within the shown frequency region. The evanescent bands at $\omega a /(2 \pi c) \approx 0.73(\star)$ do not exhibit a fork within the decay rate plot and are coupling to the incident plane wave. They can henceforth act as a communication channel between the incident wave and the weakly coupling propagating bands at the same frequency ( $\circ$ ). The presence of this communication channel explains why the reflectance rates are small in the whole frequency range despite the absence of strongly coupling and propagating bands in a broad region within this range: this region is marked with $(\times)$ in Figure 14C, which is reproduced from [14]. The absence of such a communication channel in the [100] and [110] directions explains the high reflectivity there; $c f$. the regions marked with $(\star)$ and $(\odot)$. 


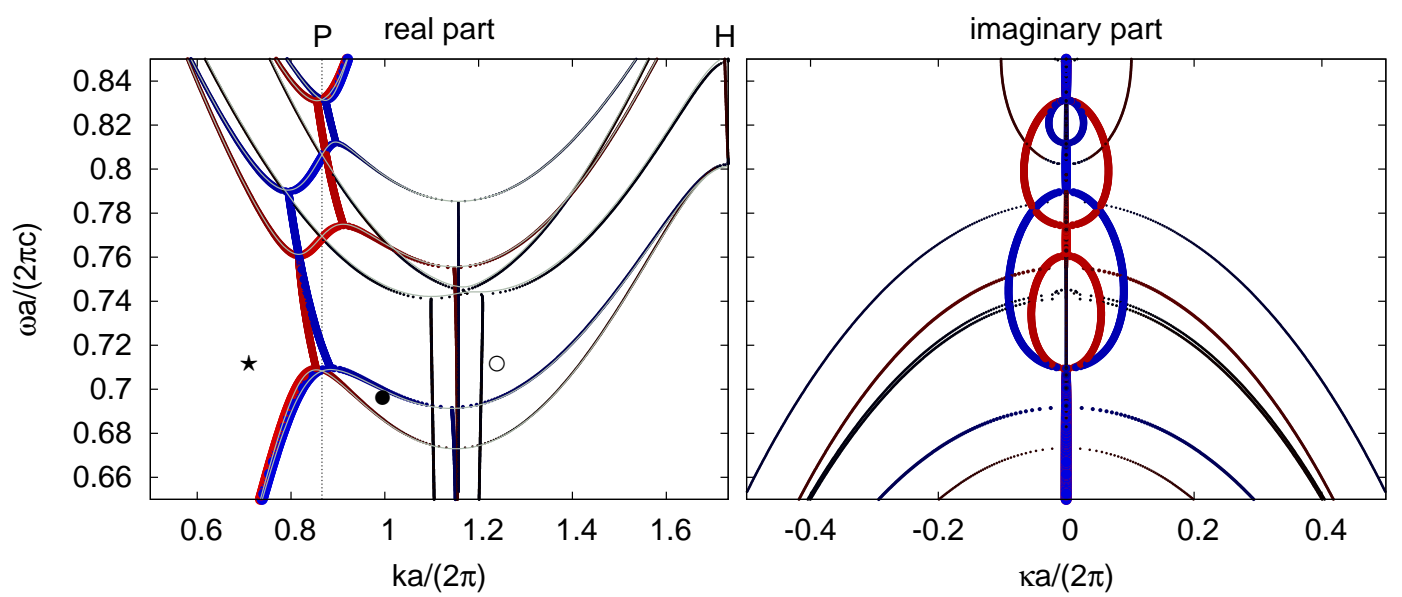

Figure 13. CBS diagram of the single gyroid photonic crystal along the [111] direction. The bands with a very large slope near $k a /(2 \pi)=2 / \sqrt{3} \approx 1.15\left(\mathbf{k}=2 / 3 \times(1,1,1)^{T}\right)$ are genuine bands. They represent perturbations of the six vacuum structure bands with $\mathbf{G}_{\|} a /(2 \pi)=1 / 3 \times(-1,-1,2)^{T}$ and the two permuted vectors thereof. All six bands have an elliptical shape in the decay rate diagram, with the unperturbed vacuum semi-ellipse defined by $\kappa=\sqrt{2 / 3-k_{0}^{2}}$.

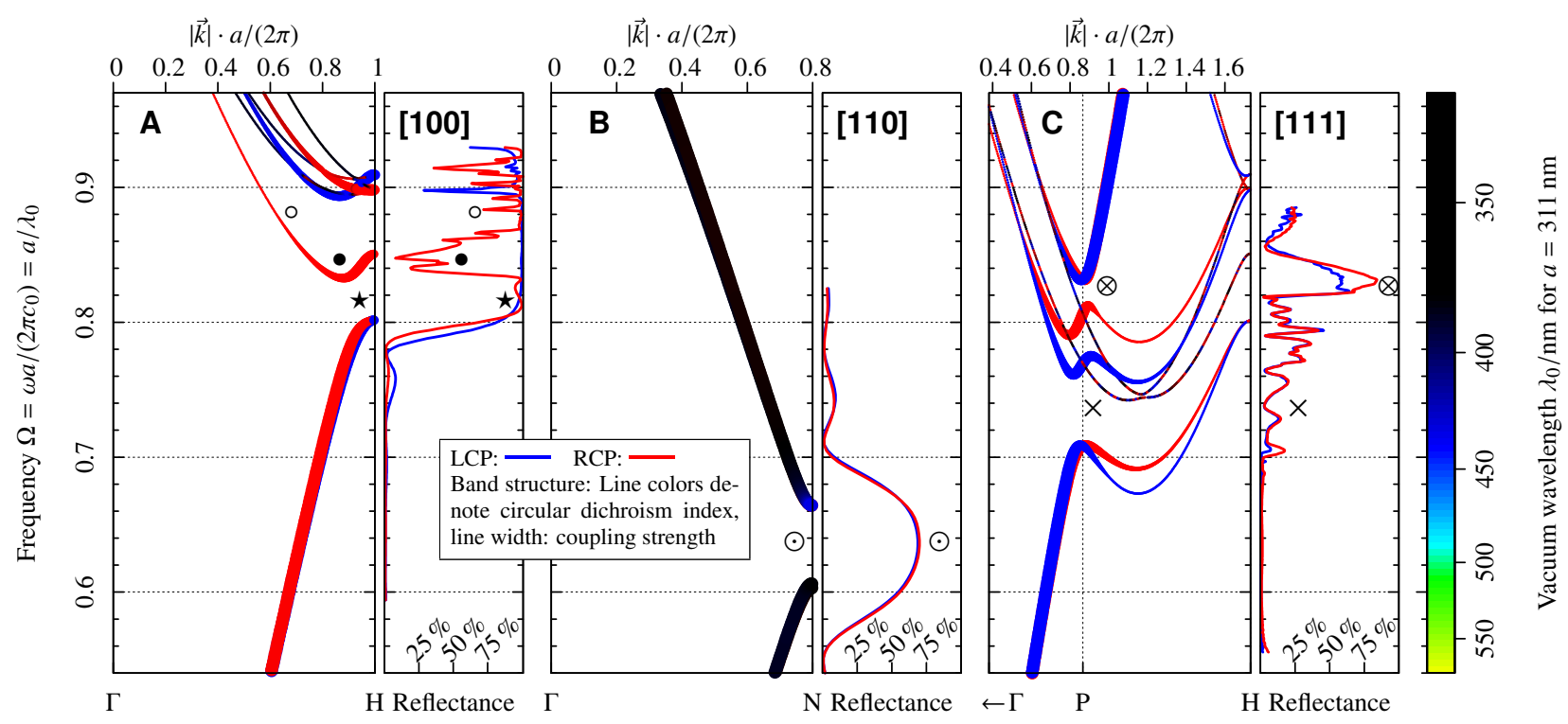

Figure 14. Direction dependence of the circular dichroism of the single gyroid structure. Circular dichroism along different crystallographic directions is quantified by the bulk band structure (left parts, coloring encodes dichroism index and line width coupling strength as defined in [5]; capital letters at the bottom denote boundaries of the Brillouin zone at high-symmetry points [53]) and by FDTD simulations of the reflectance (right parts, averaged over finite sheets of gyroid material of thickness $5.5 \cdots 6.1 \times a$ and terminations $z_{0}=1 / 30,4 / 30$ ): (A) [100] direction, (B) [110] direction, (C) [111] direction. The single gyroid is modelled by the nodal approximation (Equation (34)) with $t=-0.90$, corresponding to a volume fraction of solid chitin of $\phi \approx 20 \%$; the dielectric constant is $\varepsilon=2.4$. 


\section{Conclusions}

The relevance of complex structures for nanofabricated or self-assembled photonic and plasmonic materials is likely to increase further. The single gyroid geometry, which epitomises a complex network-like morphology, only represents the current pinnacle of this trend, with an ever-increasing plethora of geometric candidate structures and with ever-increasing abilities to fabricate structures of almost arbitrary complexity by direct writing techniques, at smaller and smaller length scales.

While direct simulation methods, such as FDTD or FEM methods, provide the most immediately accessible data for the material properties (such as transmission, reflectance or, in the case, of chiral materials, also optical activity and circular dichroism), the photonic band structure remains an invaluable tool to gain insight into the physics behind these observations. A significant limitation in this regard is the fact that methods based on the solution of Hermitian positive-definite eigenproblems (such as the one implemented in MPB) are limited to the purely dispersive modes and cannot compute the evanescent modes. Numerical methods, such as the one developed here, to calculate the full complex band structure (including evanescent modes) are needed. Furthermore, solving the actual scattering problem requires the smallest $k_{z}\left(k_{0}, \mathbf{k}_{\|}\right)$, rather than $\omega(\mathbf{k})$, yielding more numerical intricacies related to a necessary matrix inversion by an LU decomposition that requires $\mathcal{O}\left(N^{2}\right)$ computational time and memory.

We hope that the work of this article and of related previous work $[5,14,18]$ will make a substantial contribution to the informed use of complex band diagrams for the interpretation of photonic properties. This contribution is three-fold:

1. Our method provides access to the full complex band structure, including the evanescent modes (in contrast to the method implemented in MPB). In this context, note also the previous work of [13].

2. Our method calculates the band structure as a function $k_{z}\left(k_{0}, \mathbf{k}_{\|}\right)$necessary for scattering problems. While suffering from $\mathcal{O}\left(N^{2}\right)$ computational cost, we have shown that reasonable convergence can be obtained by the more accurate representation of complex geometries by a mesh representation of the interfacial surface, rather than by cubic voxel representations of the solid matrix. In this context, note also the previous work on two-dimensional Fourier transforms [35].

3. Our method is not restricted to lossless dielectric materials, but is equally applicable to materials with arbitrary values of the complex dielectric tensor $\underline{\underline{\varepsilon}}$ (including losses) and also those with magnetic permittivity $\underline{\underline{\mu}}$ and coupling tensor $\underline{\underline{\zeta}}$, all of which are not covered by the method implemented in MPB.

Algorithmic improvements to the method are desirable, e.g., to improve the convergence behaviour beyond a first-order method or to reduce the computational complexity of $\mathcal{O}\left(N^{2}\right)$. Both points could be addressed, for example, by using a Nédélec finite element basis [54] on a three-dimensional triangulation instead of the plane wave basis used here. The method in its current implementation is, however, sufficiently fast to deal with complex photonic materials, as demonstrated in Section 5.

The example of the single gyroid geometry demonstrates both the necessity to include evanescent modes for the analysis of transmission spectra and the applicability of the methods developed here. This necessity is most evident for the [111] direction, for which the transmission spectrum cannot be rationalised by consideration of the (purely dispersive) Bloch modes alone. 


\section{Acknowledgments}

Gerd E. Schröder-Turk acknowledges the hospitality and support of the Department of Applied Maths at the Australian National University in Canberra. We thank the "Engineering of Advanced Materials" cluster of excellence at the Friedrich-Alexander University Erlangen-Nuremberg for financial support. We are grateful to Johannes Hielscher for the discussion and thorough comments on the manuscript. We thank the anonymous reviewers for their very constructive criticism and for pointing us to [35].

\section{Author Contributions}

Matthias Saba developed the concept and the method described in the article, implemented its algorithmic solution, analysed and interpreted the data for the photonic crystal structures discussed here and wrote the manuscript. Gerd E. Schröder-Turk interpreted the photonic data, critically commented on the concept and method and wrote the manuscript.

\section{Conflicts of Interest}

The authors declare no conflict of interest.

\section{References}

1. John, S. Strong Localization of Photons in Certain Disordered Dielectric Superlattices. Phys. Rev. Lett. 1987, 58, 2486-2489.

2. Joannopoulos, J.; Johnson, S.; Winn, J.; Meade, R. Photonic Crystals: Molding the Flow of Light; Princeton University Press: Princeton, NY, USA, 2008.

3. Karkashadze, D.; Bogdanov, F.; Zaridze, R.; Bijamov, A.; Hafner, C.; Erni, D. Simulation of Finite Photonic Crystals Made of Biisotropic or Chiral Material. In Advances in Electromagnetics of Complex Media and Metamaterials; NATO Science Series; Zouhdi, S., Sihvola, A., Arsalane, M., Eds.; Springer: Berlin, Germany, 2002; Volume 89, pp. 175-193.

4. Lee, J.; Chan, C.T. Polarization gaps in spiral photonic crystals. Opt. Express 2005, 13, 8083-8088.

5. Saba, M.; Thiel, M.; Turner, M.; Hyde, S.T.; Gu, M.; Große-Brauckmann, K.; Neshev, D.; Mecke, K.; Schröder-Turk, G.E. Circular Dichroism in Biomimetic Cubic Chiral Nets. Phys. Rev. Lett. 2011, 106, doi:10.1103/PhysRevLett.106.103902.

6. Dolan, J.A.; Wilts, B.D.; Vignolini, S.; Baumberg, J.J.; Steiner, U.; Wilkinson, T.D. Optical Properties of Gyroid Structured Materials: From Photonic Crystals to Metamaterials. Adv. Opt. Mater. 2014, doi:10.1002/adom.201400333.

7. Lawrence, F.J.; Botten, L.C.; Dossou, K.B.; McPhedran, R.C.; Martijn de Sterke, C. A flexible Bloch mode method for computing complex band structures and impedances of two-dimensional photonic crystals. J. Appl. Phys. 2012, 111, doi:10.1063/1.3674281.

8. Dossou, K.B.; Botten, L.C. A combined three-dimensional finite element and scattering matrix method for the analysis of plane wave diffraction by bi-periodic, multilayered structures. J. Comput. Phys. 2012, 231, 6969-6989. 
9. Moharam, M.G.; Gaylord, T.K. Rigorous coupled-wave analysis of planar-grating diffraction. J. Opt. Soc. Am. 1981, 71, 811-818.

10. Botten, I.C.; Craig, M.S.; McPhedran, R.C.; Adams, J.L.; Andrewartha, J.R. The dielectric lamellar diffraction grating. Opt. Acta 1981, 28, 413-428.

11. Whittaker, D.M.; Culshaw, I.S. Scattering-matrix treatment of patterned multilayer photonic structures. Phys. Rev. B 1999, 60, 2610-2618.

12. Rumpf, R.C. Improved formulation of scattering matrices for semi-analytical methods that is consistent with convention. Prog. Electromagn. Res. B 2011, 35, 241-261.

13. Feng, C.S.; Mei, L.M.; Cai, L.Z.; Yang, X.L.; Wei, S.S.; Li, P. A plane-wave-based approach for complex photonic band structure and its applications to semi-infinite and finite system. J. Phys. D 2006, 39, doi:10.1088/0022-3727/39/20/005.

14. Saba, M.; Wilts, B.; Hielscher, J.; Schröder-Turk, G.E. Absence of circular polarisation in reflections of butterfly wing scales with chiral Gyroid structure. Mater. Today Proc. 2014, doi:10.1016/j.matpr.2014.09.023.

15. Turner, M.D.; Saba, M.; Zhang, Q.; Cumming, B.P.; Schröder-Turk, G.E.; Gu, M. Miniature chiral beamsplitter based on gyroid photonic crystals. Nat. Photon. 2013, 7, 801-805.

16. Hamming, R.W. Numerical Methods for Scientists and Engineers; Dover: New York, NY, USA, 1986.

17. Johnson, S.G.; Joannopoulos, J.D. Block-iterative frequency-domain methods for Maxwell's equations in a planewave basis. Opt. Express 2001, 8, 173-190.

18. Saba, M.; Turner, M.D.; Mecke, K.; Gu, M.; Schröder-Turk, G.E. Group theory of circular-polarization effects in chiral photonic crystals with four-fold rotation axes applied to the eight-fold intergrowth of gyroid nets. Phys. Rev. B 2013, 88, doi:10.1103/PhysRevB.88.245116.

19. Cangellaris, A.; Wright, D. Analysis of the numerical error caused by the stair-stepped approximation of a conducting boundary in FDTD simulations of electromagnetic phenomena. IEEE Trans. Antennas Propag. 1991, 39, 1518-1525.

20. Farjadpour, A.; Roundy, D.; Rodriguez, A.; Ibanescu, M.; Bermel, P.; Joannopoulos, J.D.; Johnson, S.G.; Burr, G.W. Improving accuracy by subpixel smoothing in the finite-difference time domain. Opt. Lett. 2006, 31, 2972-2974.

21. Schoen, A.H. Infinite Periodic Minimal Surfaces without Self-Intersections; Technical Note TN D-5541; NASA: Washington, DC, USA, 1970.

22. Große-Brauckmann, K. Gyroids of constant mean curvature. Exp. Math. 1997, 6, 33-50.

23. Michielsen, K.; Stavenga, D.G. Gyroid cuticular structures in butterfly wing scales: Biological photonic crystals. J. R. Soc. Interface 2008, 5, 85-94.

24. Hur, K.; Francescato, Y.; Giannini, V.; Maier, S.A.; Hennig, R.G.; Wiesner, U. Three-Dimensionally Isotropic Negative Refractive Index Materials from Block Copolymer Self-Assembled Chiral Gyroid Networks. Angew. Chem. Int. Ed. 2011, 50, 11985-11989.

25. Lu, L.; Fu, L.; Joannopoulos, J.D.; Soljačić, M. Weyl points and line nodes in gyroid photonic crystals. Nat. Photon. 2013, 7, 294-299. 
26. Vignolini, S.; Yufa, N.A.; Cunha, P.S.; Guldin, S.; Rushkin, I.; Stefik, M.; Hur, K.; Wiesner, U.; Baumberg, J.J.; Steiner, U. A 3D Optical Metamaterial Made by Self-Assembly. Adv. Mater. 2011, 24, OP23-OP27.

27. Oh, S.S.; Demetriadou, A.; Wuestner, S.; Hess, O. On the Origin of Chirality in Nanoplasmonic Gyroid Metamaterials. Adv. Mater. 2012, 25, 612-617.

28. Oskooi, A.F.; Roundy, D.; Ibanescu, M.; Bermel, P.; Joannopoulos, J.; Johnson, S.G. MeEP: A flexible free-software package for electromagnetic simulations by the FDTD method. Comput. Phys. Commun. 2010, 181, 687-702.

29. Condon, E.U. Theories of Optical Rotatory Power. Rev. Mod. Phys. 1937, 9, 432-457.

30. Sihvola, A.H. Electromagnetic Modeling of Bi-Isotropic Media. PIER 1994, 9, 45-86.

31. Jackson, J. Classical Electrodynamics; John Wiley \& Sons: Hoboken, NJ, USA, 1975.

32. Spivak, M. Calculus on Manifolds: A Modern Approach to Classical Theorems of Advanced Calculus; Westview Press: Boulder, CO, USA, 1971.

33. Boffi, D.; Fortin, M.; Brezzi, F. Mixed Finite Element Methods and Applications; Springer: Berlin, Germany, 2013.

34. Dossou, K.; Fontaine, M. A high order isoparametric finite element method for the computation of waveguide modes. Comput. Methods Appl. Mech. Eng. 2005, 194, 837-858.

35. Sorets, E. Fast Fourier Transforms of Piecewise Constant Functions. J. Comput. Phys. 1995, 116, 369-379.

36. Schröder-Turk, G.E.; Mickel, W.; Kapfer, S.C.; Schaller, F.M.; Breidenbach, B.; Hug, D.; Mecke, K. Minkowski tensors of anisotropic spatial structure. New J. Phys. 2013, 15, doi:10.1088/1367-2630/15/8/083028.

37. Cromwell, P.R. Polyhedra; Cambridge University Press: Cambridge , UK, 1997.

38. Lehoucq, R.B.; Sorensen, D.C.; Yang, C. ARPACK Users Guide: Solution of Large Scale Eigenvalue Problems by Implicitly Restarted Arnoldi Methods; SIAM: Philadelphia, PA, USA, 1998.

39. Press, W.H.; Teukolsky, S.A.; Vetterling, W.T.; Flannery, B.P. Numerical Recipes in C: The Art of Scientific Computing, 2nd ed.; Cambridge University Press: New York, NY, USA, 1992.

40. Rayleigh, L. On the Dynamical Theory of Gratings. Proc. R. Soc. A 1907, 79, 399-416.

41. Botten, L.C.; McPhedran, R.C.; Byrne, M.A.; Asatryan, A.A.; Nicorovici, N.A.; Norton, A.H.; de Sterke, C.M. The Modelling of Fano Resonances in Photonic Crystal Slabs. Available online: http://www.opticsinfobase.org/abstract.cfm?URI=META-2006-ThD15 (accessed on 2 January 2015).

42. Cao, Y.; Li, J. Complete band gaps in one-dimensional photonic crystals with negative refraction arising from strong chirality. Phys. Rev. B 2014, 89, doi:10.1103/PhysRevB.89.115420.

43. Brakke, K.A. The surface evolver. Exp. Math. 1992, 1, 141-165.

44. Thomson, W. On the division of space with minimum partitional area. Philos. Mag. 1887, 24, 503-514.

45. Michielsen, K.; Kole, J.S. Photonic band gaps in materials with triply periodic surfaces and related tubular structures. Phys. Rev. B 2003, 68, doi:10.1103/PhysRevB.68.115107. 
46. Schwarz, H.A. Gesammelte Mathematische Abhandlungen; Springer: Berlin, Germany, 1933; Volume 2.

47. Aspnes, D.E. Local field effects and effective medium theory: A microscopic perspective. Am. J. Phys. 1982, 50, 704-709.

48. Hyde, S.T.; O'Keeffe, M.; Proserpio, D.M. A short history of an elusive yet ubiquitous structure in chemistry, materials and mathematics. Angew. Chem. Int. Ed. 2008, 47, 7996-8000.

49. Saranathan, V.; Osuji, C.; Mochrie, S.; Noh, H.; Narayanan, S.; Sandy, A.; Dufresne, E.; Prum, R. Structure, function, and self-assembly of single network gyroid (I4132) photonic crystals in butterfly wing scales. Proc. Natl. Acad. Sci. USA 2010, 107, 11676-11681.

50. Schröder-Turk, G.; Wickham, S.; Averdunk, H.; Large, M.; Poladian, L.; Brink, F.; Fitz Gerald, J.; Hyde, S.T. The chiral structure of porous chitin within the wing-scales of Callophrys rubi. J. Struct. Biol. 2011, 174, 290-295.

51. Kato, T. Perturbation Theory for Linear Operators; Springer: Berlin, Germany, 1995.

52. Nachtrab, S.; Kapfer, S.C.; Rietzel, D.; Drummer, D.; Madadi, M.; Arns, C.H.; Kraynik, A.M.; Schröder-Turk, G.E.; Mecke, K. Tuning Elasticity of Open-Cell Solid Foams and Bone Scaffolds via Randomized Vertex Connectivity. Adv. Eng. Mater. 2012, 14, 120-124.

53. Ashcroft, N.W.; Mermin, N.D. Solid State Physics; Saunders College: New York, USA, 1976.

54. Nédélec, J. A new family of mixed finite elements in $\mathbb{R}^{3}$. Numer. Math. 1986, 50, 57-81.

(c) 2015 by the authors; licensee MDPI, Basel, Switzerland. This article is an open access article distributed under the terms and conditions of the Creative Commons Attribution license (http://creativecommons.org/licenses/by/4.0/). 